University of Warwick institutional repository: http://go.warwick.ac.uk/wrap This paper is made available online in accordance with publisher policies. Please scroll down to view the document itself. Please refer to the repository record for this item and our policy information available from the repository home page for further information.

To see the final version of this paper please visit the publisher's website. Access to the published version may require a subscription.

Author(s): P. J. THOMAS and P. F. LINDEN

Article Title: Rotating gravity currents: small-scale and large-scale laboratory experiments and a geostrophic model Year of publication: 2007 Link to published version: http://dx.doi.org/ 10.1017/S0022112007004739

Publisher statement: None 


\title{
Rotating gravity currents: small-scale and large-scale laboratory experiments and a geostrophic model
}

\author{
P. J. THOMAS $S^{1}$ AND P. F. LINDEN ${ }^{2}$ \\ ${ }^{1}$ Fluid Dynamics Research Centre, School of Engineering, University of Warwick, \\ Coventry, CV4 7AL, UK \\ ${ }^{2}$ Department of Mechanical and Aerospace Engineering, University of California, San Diego, \\ 9500 Gilman Drive, La Jolla, CA 92093-0411, USA
}

(Received 14 December 2005 and in revised form 31 October 2006)

Laboratory experiments simulating gravity-driven coastal surface currents produced by estuarine fresh-water discharges into the ocean are discussed. The currents are generated inside a rotating tank filled with salt water by the continuous release of buoyant fresh water from a small source at the fluid surface. The height, the width and the length of the currents are studied as a function of the background rotation rate, the volumetric discharge rate and the density difference at the source. Two complementary experimental data sets are discussed and compared with each other. One set of experiments was carried out in a tank of diameter $1 \mathrm{~m}$ on a small-scale rotating turntable. The second set of experiments was conducted at the large-scale Coriolis Facility (LEGI, Grenoble) which has a tank of diameter $13 \mathrm{~m}$. A simple geostrophic model predicting the current height, width and propagation velocity is developed. The experiments and the model are compared with each other in terms of a set of non-dimensional parameters identified in the theoretical analysis of the problem. These parameters enable the corresponding data of the large-scale and the small-scale experiments to be collapsed onto a single line. Good agreement between the model and the experiments is found.

\section{Introduction}

When estuarine water is discharged into the coastal zone, gravity-driven surface flows can be established. These flows develop as a consequence of the density difference between the buoyant estuarine fresh water and the denser, salty ocean water. For sufficiently large discharge rates, i.e. when the current exceeds length scales larger than the Rossby deformation scale, the current dynamics are affected by the Coriolis force arising from the rotation of the earth. As a result the discharged fresh water is confined to the coastal zone, where it forms a current flowing along the coast. Typical examples are, for instance, the Columbia River Plume (Hickey et al. 1998), the Delaware Coastal Current (Münchow \& Garvine 1993a, b), the Hudson River Plume (e.g. Bowman \& Iverson 1978), the Chesapeake Bay Outflow (Rennie, Largier \& Lentz 1999), the Tsugaru (e.g. Kawasaki \& Sugimoto 1984) the Algerian Current, the East Greenland Current, the Leeuwinn Current (Chabert D'Hieres, Didelle \& Obaton 1991) and outflows from certain fjords (Griffiths \& Linden 1981).

A substantial number of publications have previously presented results from field experiments (Hogg \& Johns 1995), computational results (Blumberg, Signell \& Jenter 
1993; Batteen 1997; Boyer, Haidvogel \& Pérenne 2001) and theoretical studies (Hacker \& Linden 2002; Martin \& Lane-Serff 2005; Martin, Smeed \& Lane-Serff 2005) on the investigation of coastal currents. Similarly, a large number of experimental laboratory studies have been described in the literature (e.g. Griffiths \& Hopfinger 1983; Griffiths 1986; Davies, Jacobs \& Mofor 1993; Simpson 1997; Thomas \& Linden 1998; Boyer et al. 2001; Avicola \& Huq 2002; Avicola \& Huq 2003a, b; Lentz \& Helfrich 2002; Rivas, Velasco Fuentes \& Ochoa 2005; Horner-Devine et al. 2006).

Davies et al. (1993) and, more recently, Lentz \& Helfrich (2002) derived scaling relations for the current width, depth and velocity as a function of the governing independent parameters. However, these relations were based on scaling arguments and, hence, did not yield the constants of proportionality required for comparison with experiment. Lentz \& Helfrich (2002) stated that these constants had to be inferred from the experimental data. We will derive a complete analytical description of the flow, under the assumption of geostrophy and zero potential vorticity, which readily yields values for the constants of proportionality. The expressions that we obtain are similar to those of Avicola \& Huq (2002), who derived a model based on the assumption that the frontal dynamics are those of a Margules front. Their results will be discussed in more detail after we have presented our model.

The predictions of the geostrophic model derived in the present paper will be compared with the experimental data obtained in small-scale and large-scale experiments. Comparison between experiment and theory is facilitated by a set of non-dimensional parameters identified in the theoretical analysis of the flow. This comparison will reveal that these parameters enable the corresponding data from small-scale and large-scale experiments to be collapsed and that the experiments agree well with our simple geostrophic model.

\section{Experimental set-up and techniques}

Two complementary sets of experiments, using different experimental facilities, were carried out. The two facilities had substantially different spatial scales, enabling the investigation of a wide range of the independent experimental parameters. The smallscale laboratory experiments were performed on a rotating turntable supporting a fluid-filled tank with diameter about $1 \mathrm{~m}$. The large-scale experiments were conducted at the Coriolis Facility (LEGI, Grenoble, France) using the $13 \mathrm{~m}$ rotating basin.

The procedures and techniques employed during the small-scale experiments are described in detail below. The experimental set-up at the Coriolis Facility was designed to mirror the small-scale study on a larger scale. We include additional comments on the techniques used in the large-scale experiments to highlight the differences from the small-scale study.

\subsection{Small-scale experiments}

The goal of the experiments was to simulate estuarine discharges of buoyant fresh water into an environment of salty, denser, ocean water. The arrangement of the experimental small-scale facility is shown in figure 1.

The experiments were carried out in a transparent circular acrylic tank with radius $R_{S}=0.4475 \pm 0.0005 \mathrm{~m}$. The tank was placed on a rotating turntable to simulate the rotation of the earth and introduce Coriolis effects on the flow. The rotation rate, $\Omega$, of the turntable was constant for each experiment. A total of 66 small-scale experiments were performed. The range over which $\Omega$ was varied and other relevant experimental parameters are summarized in table 1 . 


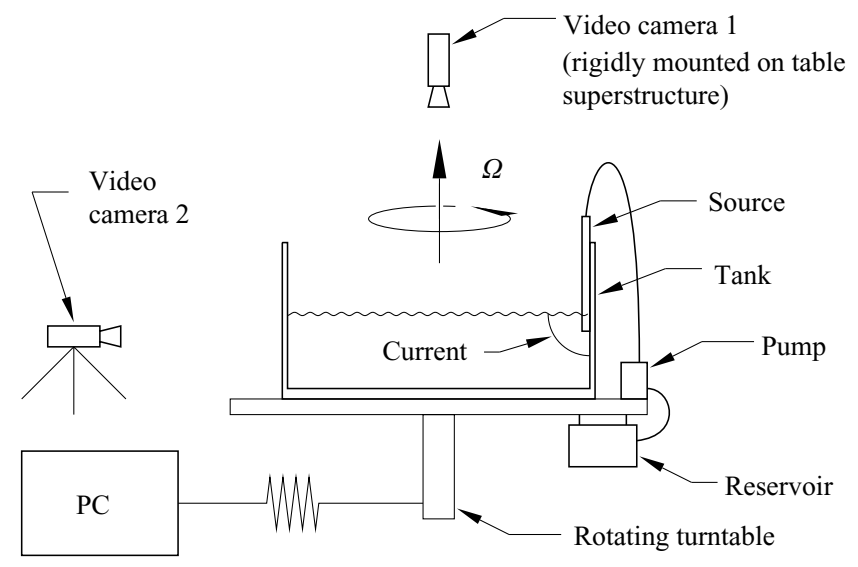

FIGURE 1. The small-scale experimental set-up.

\begin{tabular}{|c|c|c|}
\hline & Small-scale experiments & Large-scale experiments \\
\hline $\begin{array}{l}\text { Radius } \\
\text { Rotation rate } \Omega \\
\text { Flow rate } q_{0} \\
\text { Reduced grav. accel. } g^{\prime} \\
I, \text { see }(3.23)\end{array}$ & $\begin{array}{c}R_{S}=0.4475 \mathrm{~m} \\
0.5 \mathrm{rad} \mathrm{s}^{-1} \leqslant \Omega \leqslant 2.5 \mathrm{rad} \mathrm{s}^{-1} \\
3.18 \mathrm{~cm}^{3} \mathrm{~s}^{-1} \leqslant q_{0} \leqslant 28.2 \mathrm{~cm}^{3} \mathrm{~s}^{-1} \\
1.9 \mathrm{~cm} \mathrm{~s}^{-2} \leqslant g^{\prime} \leqslant 86 \mathrm{~cm} \mathrm{~s}^{-2} \\
0.0788 \leqslant I \leqslant 3.317\end{array}$ & $\begin{array}{c}R_{L}=6.5 \mathrm{~m} \\
0.0196 \mathrm{rad} \mathrm{s}^{-1} \leqslant \Omega \leqslant 0.157 \mathrm{rad} \mathrm{s}^{-1} \\
200 \mathrm{~cm}^{3} \mathrm{~s}^{-1} \leqslant q_{0} \leqslant 3972 \mathrm{~cm}^{3} \mathrm{~s}^{-1} \\
3.5 \mathrm{~cm} \mathrm{~s}^{-2} \leqslant g^{\prime} \leqslant 30.2 \mathrm{~cm} \mathrm{~s}^{-2} \\
0.00732 \leqslant I \leqslant 0.388\end{array}$ \\
\hline
\end{tabular}

The tank was filled with salt water of density $\rho_{2}$ representing the ocean water. Fresh water of density $\rho_{1}$, with $\rho_{1}<\rho_{2}$, was released from a small source mounted at the wall of the tank to simulate estuarine discharges. Fluid was released continuously and with a constant volumetric discharge rate, $q_{0}$ (see table 1 ). The density difference between the fresh water and the salt water is characterized in terms of the reduced gravitational acceleration, $g^{\prime}$, defined by

$$
g^{\prime}=\frac{\rho_{2}-\rho_{1}}{\rho_{1}} g
$$

with $g=981 \mathrm{~cm} \mathrm{~s}^{-2}$.

The depth of the salt water inside the tank was typically $10-15 \mathrm{~cm}$. It was verified experimentally that this depth was sufficient to ensure that the current properties were not measurably affected by the depth of the ambient salt water. The source from which the fresh fluid was released was adjusted to be level with the surface of the dense salt water. The source outlet was circular and of diameter $1 \mathrm{~cm}$. The fluid was supplied to the source by means of a calibrated peristaltic pump from a reservoir mounted on the turntable. The fluid was discharged vertically upwards, rather than horizontally as would be the case in the natural environment. The purpose of the vertical discharge direction was to minimize momentum-flux effects and the mixing of fresh water and salt water near the source. The salt water inside the tank was in solid-body rotation prior to the start of the release of fluid from the source. For 
visualization purposes the fresh water discharging at the source was dyed with food colouring while the ambient salt water was clear.

The experiments were filmed with two video cameras simultaneously. One camera was rigidly mounted on the superstructure of the rotating turntable and filmed the current from above the centre of the tank (figure 1). The second camera was positioned next to the turntable and filmed the flow through the transparent sidewall of the acrylic tank. This camera thus scanned the current height at the wall of the tank once per turntable revolution.

\subsection{Large-scale experiments}

The Coriolis Facility of the Laboratoire des Ecoulements Géophysiques et Industriels (LEGI) at Grenoble, France, is the world's largest rotating platform for the simulation of oceanographic flows in the laboratory. The total diameter of the platform is $14 \mathrm{~m}$ and the radius of the steel basin supported by the facility is $R_{L}=6.5 \mathrm{~m}$. The Coriolis Facility enables experiments which are to leading order non-viscous and dominated by background rotation, i.e. the Reynolds number is large while the Rossby number is small. Details of the technical specifications of the facility can be found at http://www.coriolis-legi.org.

We carried out 34 large-scale experiments. The ranges over which the experimental parameters were varied during these experiments are included in table 1. Each experiment on the large-scale facility was filmed with seven video cameras simultaneously. These cameras were mounted at various appropriate locations on the rotating platform. Some cameras monitored sections of the tank from a few metres vertically above the fluid surface. Others were positioned at windows in the sidewall of the tank. Additionally several conductivity probes and ultrasonic profilers were mounted on the facility to measure the internal velocity and density structure of the currents.

The salt water in the tank was approximately $80 \mathrm{~cm}$ deep. Fresh fluid was supplied to the source mounted at the wall of the tank from storage reservoirs housed in the basement of the laboratory. The source and the supply system were made from commercially available drainage pipes of approximate diameter $15 \mathrm{~cm}$.

\section{Theoretical description of the problem}

Figure 2 illustrates the geometry used to describe the motion of the current along the wall of the tank. The curvature of the circular wall is neglected, and a Cartesian coordinate system $x, y, z$ is introduced as indicated in the figure. The origin of the coordinate system coincides with the outlet of the source from which the buoyant, fresh, fluid discharges. It is assumed that the velocity components in the $y$ - and $z$-directions are negligible in comparison to the velocity component $u$ in the $x$ direction. Further, we neglect all variations in the $x$-coordinate direction so that $\boldsymbol{u}=(u(y, z), 0,0)$.

The pressure, $p$, is given by the hydrostatic relation

$$
p=\left\{\begin{array}{lr}
g \rho_{1}(\eta-z), & -h<z<\eta, \\
g \rho_{1}(h+\eta)-g \rho_{2}(z+h), & z<-h,
\end{array}\right.
$$

where $\eta=\eta(y)$ represents the free-surface elevation above $z=0$ and the pressure at the free surface $z=\eta$ is taken as $p=0$.

We consider that case for which the ambient fluid is infinitely deep, so that there is no interaction between the current and the bottom of the tank. We also assume that 


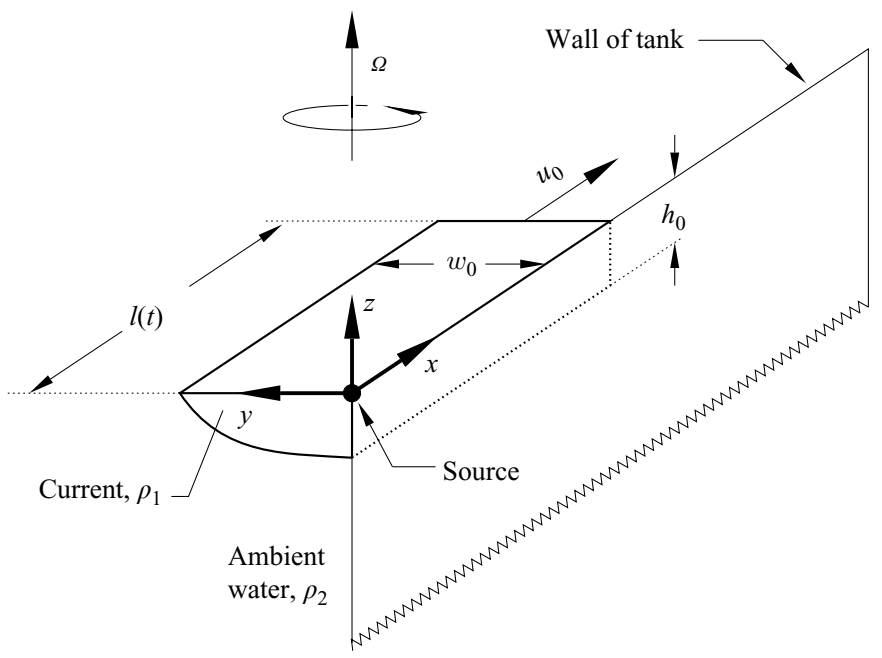

FIGURE 2. The nomenclature employed to develop the theoretical model for the current.

the current is in geostrophic balance,

$$
2 \rho_{1} \Omega u=-\frac{\partial p}{\partial y},
$$

where $\Omega$ is the rotation rate. From (3.1) one obtains

$$
\frac{\partial p}{\partial y}=\left\{\begin{array}{lr}
g \rho_{1} \frac{\partial \eta}{\partial y}, & -h<z<\eta, \\
g\left(\rho_{1}-\rho_{2}\right) \frac{\partial h}{\partial y}+g \rho_{1} \frac{\partial \eta}{\partial y}, & z<-h .
\end{array}\right.
$$

In the infinitely deep lower layer, i.e. for $z<-h$, there is no flow. Hence there the pressure gradient $\partial p / \partial y=0$, and (3.3) for the lower layer yields

$$
\rho_{1} \frac{\partial \eta}{\partial y}=\left(\rho_{2}-\rho_{1}\right) \frac{\partial h}{\partial y} .
$$

Comparison of (3.4) with the pressure gradient $\partial p / \partial y$ for $-h<z<\eta$ in (3.3) then reveals that, in the current,

Substituting (3.5) into (3.2) gives

$$
\frac{\partial p}{\partial y}=\left(\rho_{2}-\rho_{1}\right) g \frac{\partial h}{\partial y .} .
$$

$$
u=-\frac{g^{\prime}}{2 \Omega} \frac{\partial h}{\partial y},
$$

where $g^{\prime}$ is defined by (2.1). The potential vorticity, $q$, in the current is

$$
q=\frac{2 \Omega-\partial u / \partial y}{h} .
$$

The source is small so that, as a first approximation, $q=0$ for the discharging fluid. In a real flow, such as a river outflow, the fluid is released from a source of finite depth. However, real flows tend to reduce in depth as they leave the source. This would suggest that, in practice, the assumption of vanishing potential vorticity is justified. Note also that the results of Martin, Smeed \& Lane-Serff (2005) suggest that the 
potential vorticity does not have a major effect on the overall speed and shape of the current. With $q=0$ and (3.7), conservation of potential vorticity then implies that $\partial u / \partial y=2 \Omega$. Differentiating (3.6) with respect to $y$ and then substituting for $\partial u / \partial y$ in (3.7) gives

$$
\frac{\partial^{2} h}{\partial y^{2}}=-\frac{4 \Omega^{2}}{g^{\prime}} .
$$

Integration of (3.8) yields

$$
h=-\frac{4 \Omega^{2}}{2 g^{\prime}} y^{2}+c y+d,
$$

where $c$ and $d$ are constants. Conservation of angular momentum implies that $u=0$ at the wall $y=0$. Hence, (3.6) implies $\partial h / \partial y=0$. Thus $c=0$ and

$$
h=h_{0}-\frac{2 \Omega^{2}}{g^{\prime}} y^{2},
$$

where $h_{0}$ is the (maximum) depth at the wall. Reference to (3.8) and (3.6) shows that this parabolic depth profile (3.10) implies that the current velocity

$$
u=2 \Omega y
$$

increases linearly with distance from the wall.

The current has maximum width $w_{0}$ where the interface (3.10) intersects the fluid surface, i.e when $h\left(y=w_{0}\right)=0$. Thus (3.10) gives

$$
w_{0}=\left(\frac{g^{\prime} h_{0}}{2 \Omega^{2}}\right)^{1 / 2} \text {. }
$$

Assuming that the width and depth of the current at any downstream location remain constant in time, continuity requires that the volumetric flow rate, $q_{0}$, from the source must equal the volumetric flow rate across any cross-section of the current. Then

$$
q_{0}=\int_{0}^{w_{0}} h(y) u(y) \mathrm{d} y,
$$

is independent of $x$. Substituting (3.10) and (3.11) into (3.13), one obtains after integration

$$
q_{0}=\left(h_{0} \Omega w_{0}^{2}-\frac{\Omega^{3} w_{0}^{4}}{g^{\prime}}\right) .
$$

From (3.12) and (3.14) one finds

$$
h_{0}=\left(\frac{4 \Omega q_{0}}{g^{\prime}}\right)^{1 / 2},
$$

and then from (3.15) and (3.12)

$$
w_{0}=\left(\frac{g^{\prime} q_{0}}{\Omega^{3}}\right)^{1 / 4} .
$$

The total current volume $V$ is given by

$$
V=q_{0} t=l \int_{0}^{w_{0}} h(y) \mathrm{d} y,
$$


where $l$ represents the length of the current. Carrying out the integration using (3.10) and then introducing (3.12) and (3.15) leads to

$$
V=q_{0} t=l \frac{4 q_{0}^{3 / 4}}{3 g^{\prime 1 / 4} \Omega^{1 / 4}} .
$$

Equation (3.18) implies that the current travels at constant speed $l / t=u_{0}$ and that the length $l$ is given by

$$
l=\frac{3}{4}\left(q_{0} g^{\prime} \Omega\right)^{1 / 4} t
$$

These scalings for the width, depth and velocity of the current are the same as those given in Davies et al. (1993) and, more recently, by Lentz \& Helfrich (2002). However, since these authors used only scaling arguments they could not give explicit values for the constants in their expressions. The definition of Horner-Devine et al. (2006) for the maximum depth in a geostrophic current is identical with the current depth obtained from our model in (3.15). The model of Avicola \& Huq (2002), which is based on the assumption that the frontal dynamics are that of a Margules front, also yields the same current depth as that in (3.15). In terms of our nomenclature the current width derived by Avicola \& Huq is $w_{0}=(1 / \sqrt{2})\left(g^{\prime} q_{0} / \Omega^{3}\right)^{1 / 4}$ and this value is thus smaller by a factor $1 / \sqrt{2}=0.707$ than that found from (3.16). From (3.19) one sees that the constant propagation speed of the current head in our model is $u_{0}=l / t=\frac{3}{4}\left(q_{0} g^{\prime} \Omega\right)^{1 / 4}$. In comparison, the speed of the current head found by Avicola \& Huq is $u_{0}=\sqrt{2}\left(q_{0} g^{\prime} \Omega\right)^{1 / 4}$. Thus, their current speed is higher by a factor $\sqrt{2} /(3 / 4)=1.89$ than that predicted by the present model.

The results above can be conveniently expressed in dimensionless form. We use the non-dimensional time $T=\Omega t$ and non-dimensionalize lengths by $w_{0}=\left(g^{\prime} q_{0} / \Omega^{3}\right)^{1 / 4}$. The scale $w_{0}$ is equivalent to the usual Rossby deformation scale, $\sqrt{g^{\prime} h_{0}} / \Omega$, for the flow based on the flow rate in the current. The deformation scale corresponds to the usual adjustment length based on potential-vorticity conservation. This can be seen as follows. Solving (3.15) for $q_{0}$, substituting this expression into (3.19) and then solving for $l / t=u_{0}$ shows that $u_{0}$ scales as $\sqrt{g^{\prime} h_{0}}$. Equation (3.12) immediately reveals the usual adjustment scaling $w_{0} \propto \sqrt{g^{\prime} h_{0}} / \Omega$. Together with $q_{0} \propto u_{0} h_{0} w_{0}$ the two scalings for $u_{0}$ and $w_{0}$ imply $q_{0} \propto \sqrt{g^{\prime} h_{0}} h_{0}\left(\sqrt{g^{\prime} h_{0}} / \Omega\right)$. By substituting $h_{0}$ in this expression by means of (3.15), rearranging and comparing with (3.16) one sees that $\sqrt{g^{\prime} h_{0}} / \Omega \propto\left(g^{\prime} q_{0} / \Omega^{3}\right)^{1 / 4}=w_{0}$. Hence $w_{0}$ is equivalent to the Rossby deformation radius based on the depth of the current.

Using capital letters to denote non-dimensional variables, (3.19) is written as

$$
L=\frac{3}{4} T \text {. }
$$

The dimensionless width is then given by

$$
W=1
$$

and the dimensionless depth is

$$
H=2 I^{5 / 4} \text {. }
$$

Reference to (3.15) and (3.16), or to (3.22) with $H=h_{0} / w_{0}$, reveals that the quantity

$$
I=\frac{\Omega q_{0}^{1 / 5}}{g^{\prime 3 / 5}},
$$

is a dimensionless parameter that describes the aspect ratio, $h_{0} / w_{0}$, of the current. Note that introducing the Coriolis parameter, $f=2 \Omega$, into our final expressions 
(3.15), (3.16) and (3.19) for the current depth, width and the length does not alter the form of the non-dimensional current width in (3.21). Neither does it affect the non-dimensional current depth given by (3.22) as long the definition of $I$ in (3.23) remains unchanged. In (3.20), which gives the non-dimensional current length, the time $T=\Omega t$ would be replaced by $f t$.

One can define a Rossby number $R o$ as

$$
R o=\frac{u_{0}}{\Omega w_{0}},
$$

where $u_{0}=l / t$ from (3.19), and a Froude number $\mathrm{Fr}$ as

$$
F r=\frac{u_{0}}{\sqrt{g^{\prime} h_{0}}} .
$$

Using the expressions (3.15) for $h_{0},(3.16)$ for $w_{0}$ and (3.19) for $u_{0}$ one finds

$$
R o=\frac{3}{4}=0.75
$$

and

$$
F r=\frac{3}{2^{5 / 2}}=0.5303
$$

The ratio of body forces and Coriolis forces is expressed by the ratio of the Rossby number and the Froude number and, with (3.26) and (3.27), one finds that

$$
\frac{R o}{F r}=\frac{2^{5 / 2}}{4}=\sqrt{2} .
$$

If one uses the expressions of Avicola \& Huq (2002) for the current width, depth and propagation velocity to calculate the Rossby number, the Froude number and the ratio of both, corresponding to the above expressions (3.26), (3.27) and (3.28), one finds $R o=2, F r=1$ and, consequently, $R o / F r=2$.

The current velocity $u_{0}=l / t$ from (3.19) together with (3.15) can also be used to define a Reynolds number $\operatorname{Re}\left(w_{0}, u_{0}\right)$

$$
R e=\frac{w_{0} u_{0}}{v}=\frac{3 q_{0}{ }^{1 / 2} g^{\prime 1 / 2}}{4 \Omega^{1 / 2} v} .
$$

Finally one can define an Ekman number $E k\left(h_{0}\right)$ as

$$
E k\left(h_{0}\right)=\frac{\nu}{f h_{0}{ }^{2}}=\frac{\nu g^{\prime}}{2 f^{2} q_{0}}=\frac{\nu g^{\prime}}{8 \Omega^{2} q_{0}} .
$$

Using the values given in table 1 together with $v=0.01 \mathrm{~cm}^{2} \mathrm{~s}^{-1}$ for the kinematic viscosity of water, the small-scale experiments turn out to have Reynolds numbers in the range $100 \leqslant \operatorname{Re}\left(w_{0}, u_{0}\right) \leqslant 5200$ while the corresponding range for the large-scale experiments is $5000 \leqslant \operatorname{Re}\left(w_{0}, u_{0}\right) \leqslant 185000$. Similarly, the small-scale experiments have Ekman numbers in the range $1.34 \times 10^{-5} \leqslant E k\left(h_{0}\right) \leqslant 1.34 \times 10^{-1}$ while the large-scale experiments have values in the range $4.47 \times 10^{-5} \leqslant E k\left(h_{0}\right) \leqslant 4.91 \times 10^{-1}$.

Finally, note that a comparison of (3.23) and (3.30) reveals that highest values of $I$ in our inviscid model always correspond to the lowest values of $E k$ and vice versa. We expect that our inviscid model will describe the experimental data better when viscosity effects are small, i.e. when the Ekman number is small or, equivalently, when $I$ is large. In particular, one sees from (3.22) that currents are shallow when $I$ is small. This means that the assumption that the currents are in geostrophic balance will then no longer hold and our scaling relations will break down. 


\section{Experimental results}

\subsection{Introductory remarks}

Figure 3(a-c) shows flow visualizations that are typical examples of currents observed in the small-scale facility for low, intermediate and high values of $I$. The pictures were obtained with the corotating camera 1 (figure 1) and show the currents viewed from above the circular tank, looking vertically downwards onto the fluid surface. The dyed current fluid appears dark in the pictures. The location of the source, where the buoyant current fluid is ejected, is indicated in figure $3(a)$ and it is the same in figures $3(b, c)$. The turntable rotates anticlockwise. The currents propagate cyclonically, i.e. also anticlockwise, around the circumference of the tank. The calibration grid visible in each picture was attached to the table top underneath the transparent bottom of the tank. The angle of each grid sector is 0.1 radians and the separation between two successive calibration circles is $5 \mathrm{~cm}$.

\subsubsection{Qualitative aspects of the current shape: small-scale currents}

A comparison of figures $3(a-c)$ reveals that the currents look qualitatively different for different values of $I$. The current shapes shown are characteristic for the different values of $I$. After some experience, it is possible to estimate the value of $I$ for any particular current by simple inspection of a flow visualization. Recall that, because of (3.23) and (3.30), the lowest values of $I$ correspond to the highest values of $E k$ and vice versa. However, it is the currents, such as the one shown in figure $3(c)$, having a low $E k$ (large $I$ ) value which are most closely governed by geostrophy. For such currents, the flow in the initial stage looks similar to the currents in figures $3(a)$ and $3(b)$. However, at some point the frontal portion of the currents separates from the source and is advected downstream with the flow. As a result a current with a constant width, as predicted by our model, is left behind. We believe that it is the type of current after the frontal portion has separated that represents a current closely governed by a geostrophic balance and which is not significantly affected by viscous effects. The currents in figures $3(a)$ and $3(b)$ do not have a constant width. The reason for this is probably that the associated values of the Ekman number are too large (low I) and the currents are not sufficiently well governed by geostrophy. This will be discussed in depth in $\S 4.5$, where the data for the current width are analysed.

The observations have shown that anticyclonically (i.e. clockwise) spinning gyre is formed at the upstream end of the current in the immediate vicinity of the source. For the currents in figures $3(a)$ and $3(b)$ it was observed that the diameter of this bulge region grows continuously with time; in these figures the diameter of the bulge is about $25 \mathrm{~cm}$. Similar gyres were observed and studied experimentally by Avicola \& Huq (2003b) and Horner-Devine et al. (2006). Nof \& Pichevin (2001) investigated the issue theoretically and predicted the existence of the growing, recirculating, bulge region. As a consequence of the gyre formation some fluid is lost from the main body of the current and this must result in discrepancies with the predictions of our model. For instance, it is expected that drainage of fluid out of the main current will tend to decrease the propagation speed of the current. Hence, the current will no longer have the constant speed predicted by our model.

For the current in figure $3(c)$, with $I=2.776$, a small gyre (diameter about $3-5 \mathrm{~cm}$ ) also exists. However, for this current with a high $I$ value (low $E k$ ), the gyre diameter was observed to remain constant and so no fluid is lost from the main current. Hence, currents with low Ekman number (high I) will generally tend to be described more accurately by our model. In comparison, one may calculate from the data in 
(a)

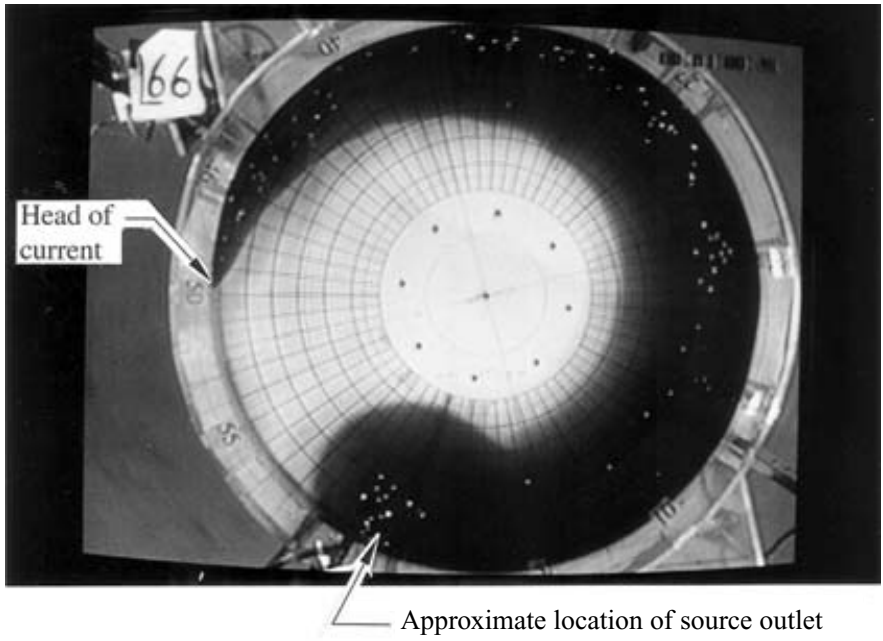

(b)

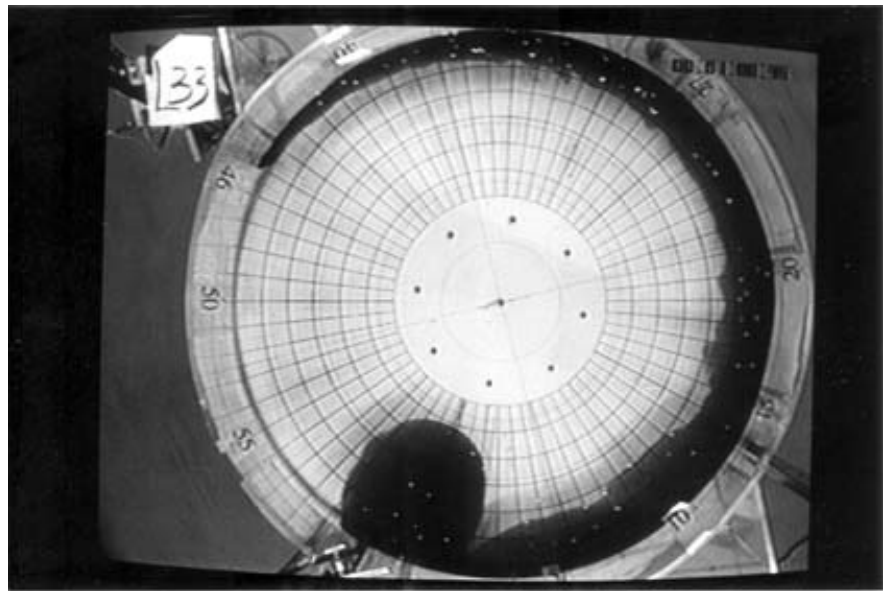

(c)

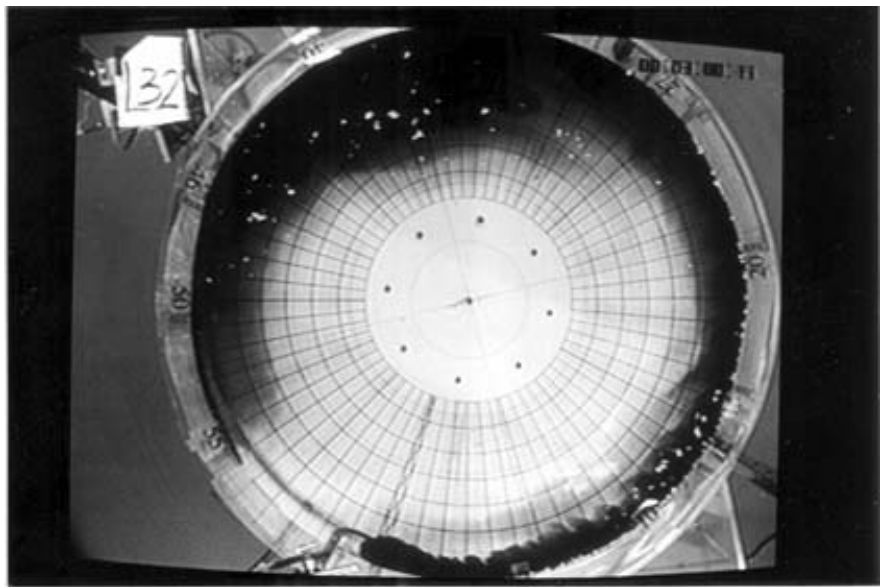

FIGURE 3. Flow visualizations showing currents (dark fluid) flowing around the wall of the circular tank in the small-scale facility. Typical shapes for different values of $I$ are shown. The turntable rotates anticlockwise. The currents also propagate anticlockwise, keeping the wall to their right. (a) Low $I$ case: $I=0.0627, t \approx 60 \mathrm{~s}, \Omega=0.5 \mathrm{rad} \mathrm{s}^{-1}, g^{\prime}=86.3 \mathrm{~cm} \mathrm{~s}^{-2}$, $q_{0}=20 \mathrm{~cm}^{3} \mathrm{~s}^{-1}$. (b) Intermediate- $I$ case: $I=0.3264, t \approx 60 \mathrm{~s}, \Omega=0.5 \mathrm{rad} \mathrm{s}^{-1}, g^{\prime}=2.26 \mathrm{~cm} \mathrm{~s}^{-2}$, $q_{0}=28.12 \mathrm{~cm}^{3} \mathrm{~s}^{-1}$. (c) High- $I$ case: $I=2.776, t \approx 180 \mathrm{~s}, \Omega=2.5 \mathrm{rad} \mathrm{s}^{-1}, g^{\prime}=2.28 \mathrm{~cm} \mathrm{~s}^{-2}$, $q_{0}=20 \mathrm{~cm}^{3} \mathrm{~s}^{-1}$. 


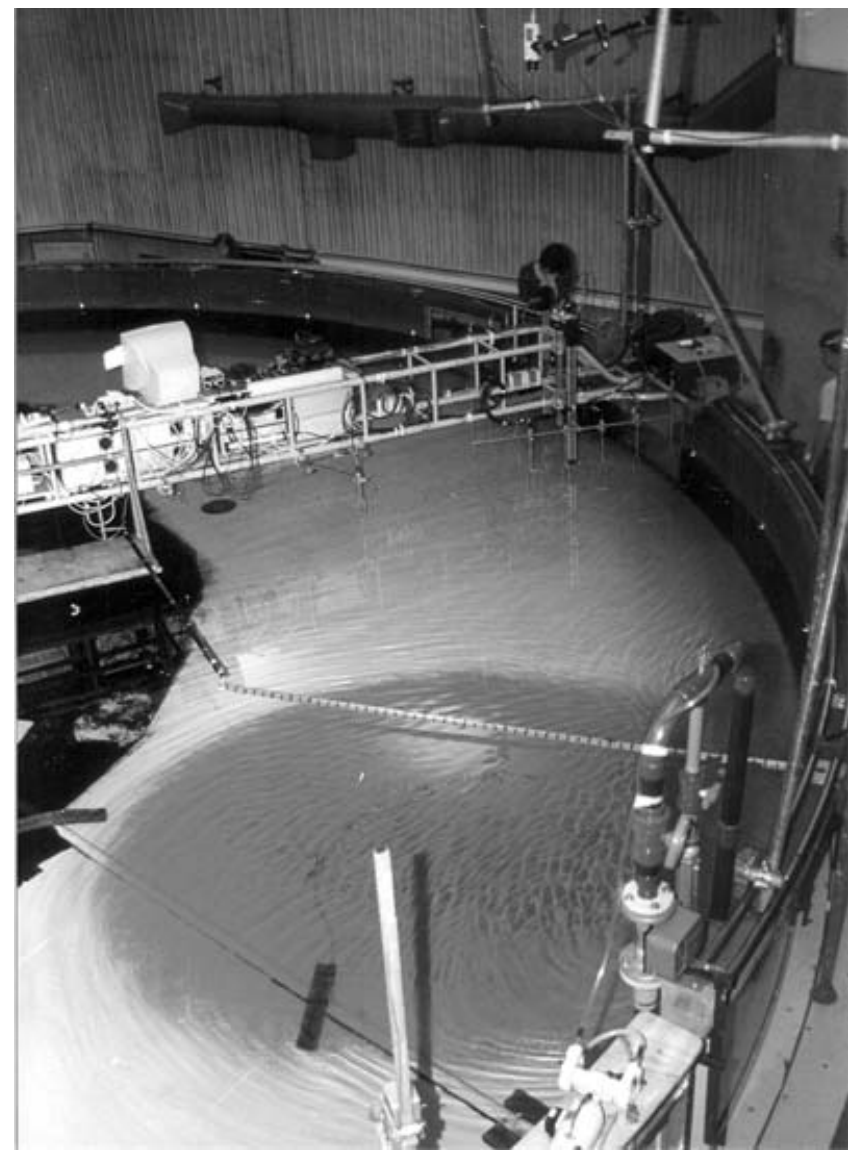

FIGURE 4. Part of a large-scale current in the Coriolis Facility.

table 1 in Avicola \& Huq (2003b) that their experiments were in the parameter regime $0.137 \leqslant I \leqslant 0.397$. Similarly, one determines $0.077 \leqslant I \leqslant 0.331$ for the experiments of Horner-Devine et al. (2006) from tables 1-3 in the appendix of their paper. Hence, all the experiments of Avicola \& Huq (2003b) and Horner-Devine et al. (2006) were within the same parameter region as our currents shown here in figures $3(a)$ and $3(b)$. None of their experiments had values as high as $I=2.776$, the parameter regime of the current in figure $3(c)$ where we observed a bulge with a constant diameter and currents of constant width. It was noted that the separation of the frontal portion of a current at high $I$, as in figure 3(c), often happens relatively late during the experiment. Hence, in order to carry out further experiments in the parameter regime of $g^{\prime}, q_{0}$ and $\Omega$ resulting in large $I$, i.e. $\sim 2-3$, it would be desirable to have a larger tank available than the $1 \mathrm{~m}$ diameter tank used in the present small-scale experiments.

\subsubsection{Qualitative aspects of the current shape: large-scale currents}

The large-scale currents observed in the Coriolis Facility look qualitatively very similar to those that develop in the small-scale facility. A photograph displaying part of a typical current during an experiment in the large-scale facility is shown in figure 4. It shows the dyed current in the region just downstream of the source. The source outlet is located at the wall of the tank, just to the right of the large circular gyre which has developed in the vicinity of the source. On the photograph the current 


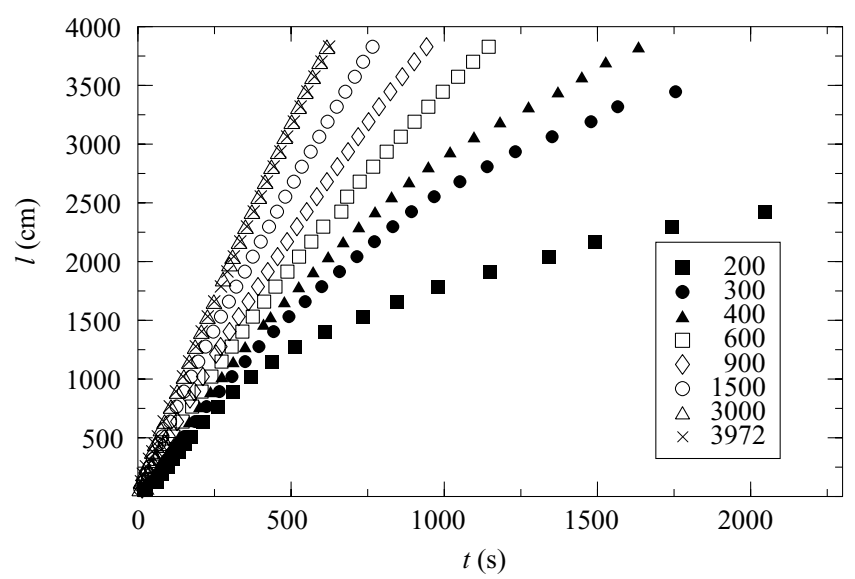

FIgURE 5. Typical raw data sets displaying the current length, 1, as a function of time, $t$, for eight large-scale experiments with different flow rates, $q_{0}$, but the same rotation rate, $\Omega=0.1571 \mathrm{rad} \mathrm{s}^{-1}$, and equal reduced gravity, $g^{\prime}=(3.57 \pm 0.15) \mathrm{cm} \mathrm{s}^{-2}$. The numbers in the key are the values of $q_{0}$ in $\mathrm{cm}^{3} \mathrm{~s}^{-1}$.

is flowing away from the viewer. The exact experimental conditions relating to the current shown are not available since they were not recorded when the picture was taken. However, figure 4 reveals that the current is relatively narrow in comparison to the gyre diameter near the source. Reference to the picture sequence for smallscale currents in figure $3(a-c)$ suggests that $I$ was of intermediate value because a comparison of the large-scale current of figure 4 shows that it looks very similar to the small-scale current with the intermediate value, $I=0.3264$, in figure $3(b)$.

\subsection{Current length}

Figure 5 shows eight typical raw-data sets for the current length $l$ as a function of time $t$. The data are from large-scale experiments with different volumetric discharge rates $q_{0}$ but equal rotation rate $\Omega=0.1571 \mathrm{rad} \mathrm{s}^{-1}$ and equal reduced gravity $g^{\prime}=(3.57 \pm 0.15) \mathrm{cm} \mathrm{s}^{-2}$. It can be seen that the currents with higher discharge rates $q_{0}$ propagate faster. Their velocity is almost constant during propagation along the entire $40 \mathrm{~m}$ circumference of the tank. Currents with lower values of $q_{0}$ initially have an approximately constant propagation velocity but gradually begin to slow down during the experiment.

Figure 6 redisplays the data of figure 5 in non-dimensional form. The solid line superposed on the figure is the theoretical prediction $L=\frac{3}{4} T$ from (3.20). The figure reveals that experiment and theory are in quite good agreement. The experimentally measured current length at early times $T$ grows faster than predicted but begins to slow down at later times. The reason for this initial velocity excess and subsequent gradual deceleration will be discussed in $\S \S 4.4 .2$ and 4.5.6.

Figures 7 and 8 display all the available data for the 66 small-scale experiments and the 34 large-scale experiments, respectively. The figures give the non-dimensional current length $L$ as a function of the non-dimensional time $T$. The solid line superposed onto each figure represents the theoretical prediction $L=\frac{3}{4} T$ from (3.20). Comparison of the two figures shows that they are very similar. In both cases experiment and theory are in good agreement. Further inspection of figures 7 and 8 reveals that a superposition of the two figures collapses the two data sets onto 


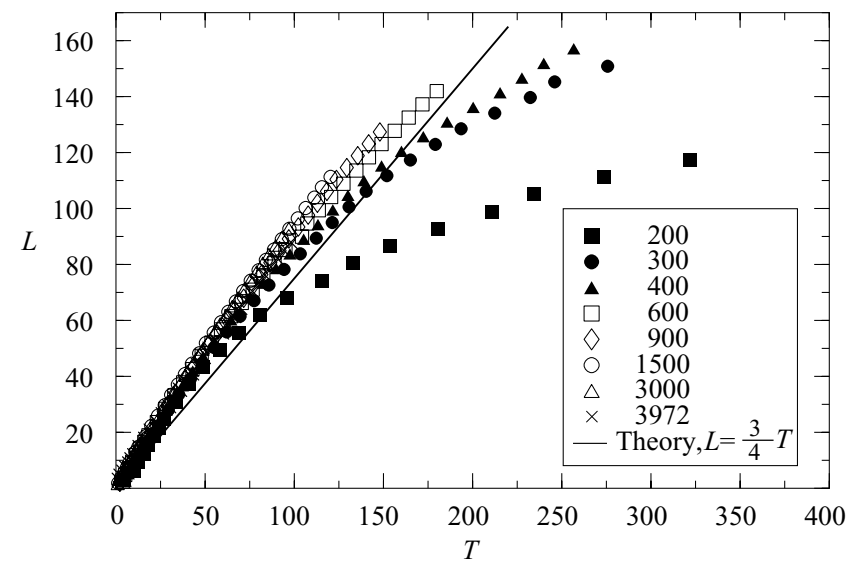

Figure 6. The data for the current length, $l$, of figure 5 in non-dimensional form: the non-dimensional current length, $L$, is shown as a function of the non-dimensional time, $T$, for eight experiments with different flow rates $q_{0}$ but equal rotation rate, $\Omega=0.1571 \mathrm{rad} \mathrm{s}^{-1}$, and equal reduced gravity, $g^{\prime}=(3.57 \pm 0.15) \mathrm{cm} \mathrm{s}^{-2}$. The numbers in the key are the values of $q_{0}$ in $\mathrm{cm}^{3} \mathrm{~s}^{-1}$.

each other. Hence the small-scale and large-scale experiments display corresponding behaviour, suggesting that the dynamics of the experiments are the same at both scales.

The dashed line superposed onto figures 7 and 8 represents the theoretical result for the current speed of Avicola \& Huq (2002). Their results have been re-expressed in our nomenclature, where the dashed line is given by $L=2 T$. This line was obtained by interpreting the current speed $c$ in expression (5) of Avicola \& Huq (2002) as $c=l / t$, so that $l=c t$ corresponds to expression (3.19) in the present paper. The current length $l$ was then non-dimensionalized using the Rossby deformation scale given in equation (4) of Avicola \& Huq (2002). Figure 7 shows that, for the small-scale experiments, our model predicts the propagation of the current head slightly better overall than the model of Avicola and Huq. For the large-scale data shown in figure 8 the prediction of Avicola and Huq seems to give slightly better agreement. However, this apparent better agreement is slightly misleading. As will be discussed in $\S 4.4 .2$, the currents have, initially, a velocity excess due to the measured along-wall depth profile. If this is taken into account then it appears justifiable to conclude that overall the experimental data for the current speed, in both the small-scale and the large-scale experiments, is in better agreement with our present model than with the model of Avicola \& Huq (2002).

\subsection{Current velocity}

Figure 9 shows examples of the ratio of the experimentally measured non-dimensional mean velocity $\overline{U_{0}^{e}}$ of the current head and the theoretically predicted current velocity $U_{0}^{t}=L / T=\frac{3}{4}$ from (3.20). The velocity data shown were obtained from the analysis of the eight experiments whose results were displayed in figures 5 and $6(\Omega=$ $\left.0.1571 \mathrm{rad} \mathrm{s}^{-1}, g^{\prime}=(3.57 \pm 0.15) \mathrm{cm} \mathrm{s}^{-2}\right)$.

It should be emphasized that $\overline{U_{0}^{e}}$ is the mean velocity given by $L / T$ and not the instantaneous current velocity $\mathrm{d} L / \mathrm{d} T$. However, the curves for the mean velocity shown in figure 9 are qualitatively similar to those one obtains for the instantaneous current velocity by calculating $\left[l\left(t_{i+1}\right)-l\left(t_{i}\right)\right] /\left(t_{i+1}-t_{i}\right)$ for a series of neighbouring data points. We have not included these curves here because the velocity data determined in this way are very sensitive to measurement errors and can fluctuate substantially. 


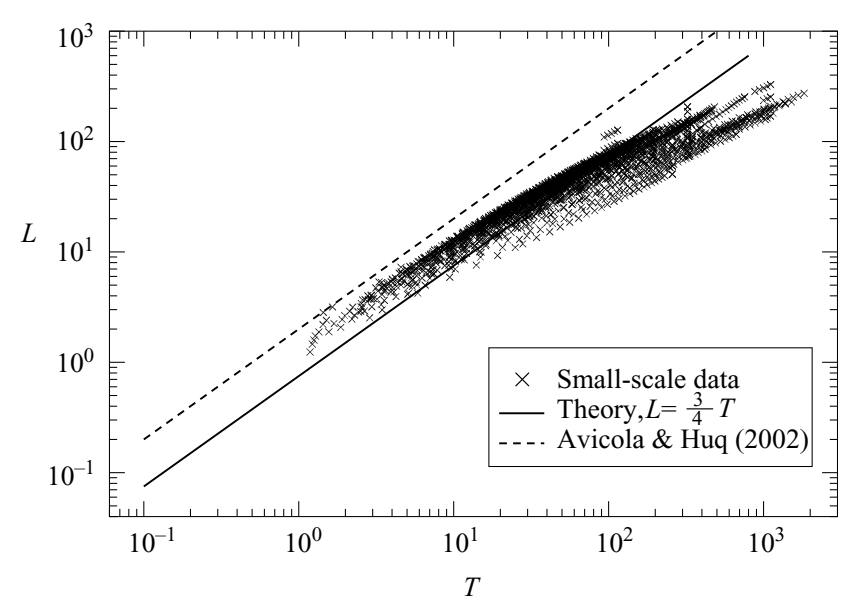

FIGURE 7. Summary of the data for the non-dimensionalized current length $L$ as a function of the non-dimensional time $T$, for the small-scale experiments. The solid line represents the theoretical prediction of (3.20).

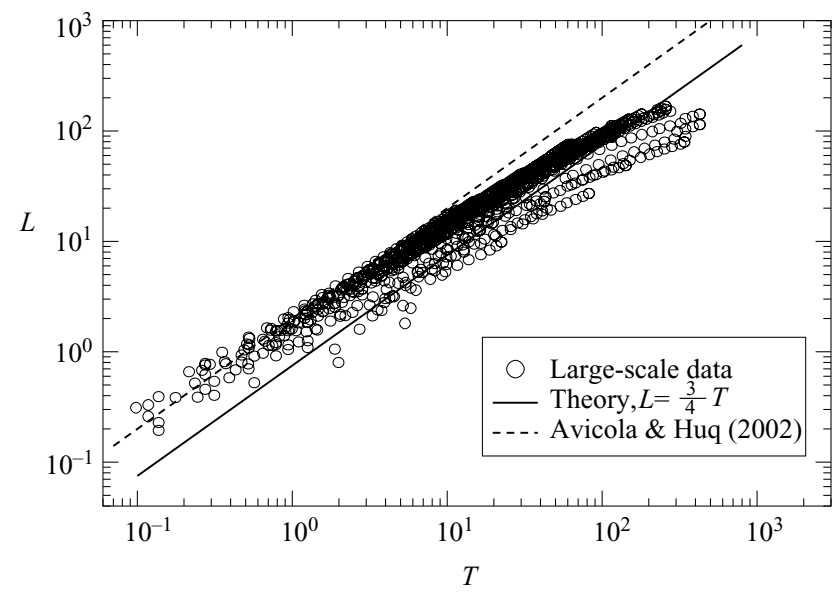

FIGURE 8. Summary of the data for the non-dimensionalized current length, $L$, as a function of the non-dimensional time, $T$, for the large-scale experiments. The solid line represents the theoretical prediction of $(3.20)$.

To see typical maximum magnitudes of these fluctuations refer to similar curves shown in Davies et al. (1993), which were obtained in this way.

Figure 9 reveals that the data for all eight experiments collapse reasonably well for times $T>25$. It can be seen how the current velocity decreases with time $T$; it is not constant as predicted by our simple geostrophic model. For times $25<T<175$ the current is faster than predicted by a factor between about 1 and 1.5 . In $\S 3$ it was noted that the current speed $U_{0}^{t}$ predicted by the model of Avicola \& Huq (2002) is higher by a factor 1.89 than the speed predicted by the present model. If the data in figure 9 were rescaled to account for this then the position of the dotted line, which indicates exact agreement between experiment and theory, would shift to $\overline{U_{0}^{e}} / U_{0}^{t}=1.89$ in the present version of the figure. Hence, the velocity predicted by Avicola \& Huq (2002) lies substantially above the measured data points displayed in figure 9 . 


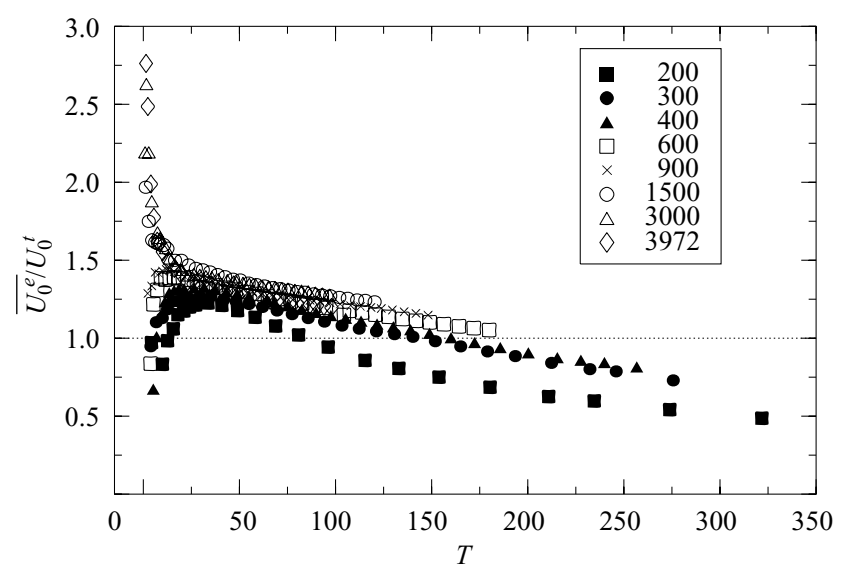

FIGURE 9. The ratio of the measured non-dimensional current mean velocity $\overline{U_{0}^{e}}$ and the predicted velocity $U_{0}^{t}=0.75$, as a function of $T$. The numbers in the key are the values of $q_{0}$ in $\mathrm{cm}^{3} \mathrm{~s}^{-1}$.

Note the behaviour displayed by the data in figure 9 during the initial phase of the experiments, for times $T<25$. Here the data points do not collapse: it can be seen that the data points for experiments with different discharge rates $q_{0}$ display qualitatively different behaviour. For $q_{0} \leqslant 900 \mathrm{~cm}^{3} \mathrm{~s}^{-1}$ the data points approach the common curve for $T \geqslant 25$ from below, while they approach it from above for $q_{0} \geqslant 1500 \mathrm{~cm}^{3} \mathrm{~s}^{-1}$. The displayed data represent experiments with flow rates 200, 300, 400,600, 900, 1500,3000 and $3972 \mathrm{~cm}^{3} \mathrm{~s}^{-1}$ and the associated values of $I$ are, respectively, 0.213 , $0.231,0.249,0.270,0.288,0.313,0.354$ and 0.387 . The change in behaviour occurs between 900 and $1500 \mathrm{~cm}^{3} \mathrm{~s}^{-1}$ and, thus, in the interval $0.288<I<0.313$. The associated ranges of the Reynolds number $\operatorname{Re}\left(w_{0}, u_{0}\right)$ and the Ekman number $E k\left(h_{0}\right)$ are $10650<\operatorname{Re}\left(w_{0}, u_{0}\right)<13943$ and $1.22 \times 10^{-4}<E k\left(h_{0}\right)<1.98 \times 10^{-4}$. However, the rotation rate $\left(\Omega=0.1571 \mathrm{rad} \mathrm{s}^{-1}\right)$ and the reduced gravity $\left(g^{\prime} \approx 3.57 \mathrm{~cm} \mathrm{~s}^{-2}\right)$ have the same values for all the experiments in figure 9. Consequently, the interval where the transition in the behaviour is observed may be different for a series of experiments having different combinations of values of $\Omega$ and $g^{\prime}$. We do not at present have data from other appropriate series of experiments available to address this issue in more detail.

\subsection{Current height}

\subsubsection{Introductory remarks}

Our model predicts that the current height $h_{0}$ at the wall of the tank (see figure 2 and (3.15)) is independent of the $x$-coordinate. Its value is equal to the current depth predicted by Avicola \& Huq (2002) and the maximum depth in a geostrophic current given in Horner-Devine et al. (2006). However, the videos recorded with camera 2 of figure 1, through the sidewall of the tank, reveal that the current height decreases between the source region and the current head. A schematic illustration of the observed height profile at the wall $(y=0)$, in the $(x, z)$-plane of figure 2 , is shown in figure 10 .

The diagram in figure 10 represents the current at an instant immediately before the end of the experiment, i.e. just before the current head returns to the source after having travelled around the entire tank. Hence, the distance between the source 


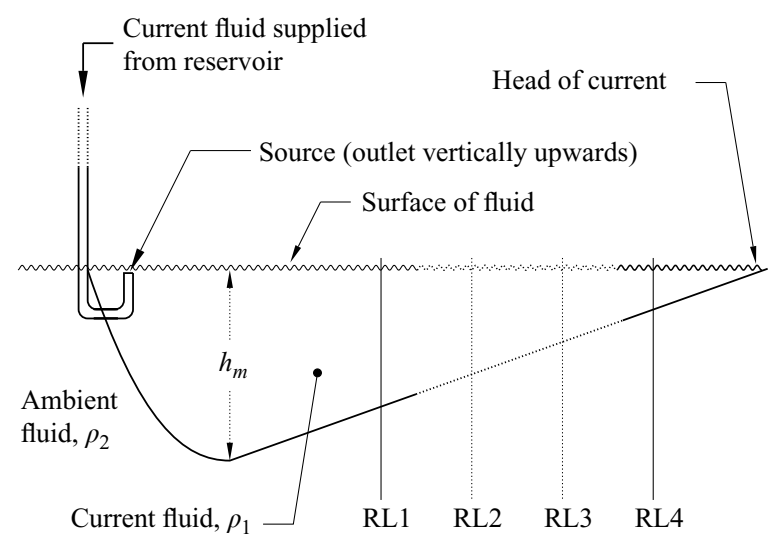

Figure 10. A side-view of the current as recorded by video camera 2, shown in figure 1 . The diagram shows how the current height changes with the along-wall distance $x$ from the source to the current head, i.e. it gives the height profile at the wall $(y=0)$ in the $(x, z)$-plane of figure 2.

and the head of the current corresponds to the circumference of the tank. It was observed that the current height has a maximum, $h_{m}$, just downstream of the source. The distance $x$ between the source and the position where the maximum height is observed is around $1-5 \mathrm{~cm}$ in the small-scale facility and around $10-50 \mathrm{~cm}$ in the largescale facility. The maximum height $h_{m}$ is, typically, in the range $0.5 \mathrm{~cm}<h_{m}<9 \mathrm{~cm}$ for the small-scale experiments and in the range $1.5 \mathrm{~cm}<h_{m}<50 \mathrm{~cm}$ for the large-scale experiments. This height $h_{m}$ was observed to be constant throughout each experiment and was used for comparison with the theoretical prediction of $h_{0}$ given by (3.15) and in non-dimensional form by (3.22).

\subsubsection{Height profile of the currents along the wall of the tank}

For the small-scale experiments we measured the maximum current height and the current height at four further reference locations along the circumference of the tank. The reference locations, identified as RL1-RL4 in figure 10, were positioned at 1.5, $2.5,3.5$ and 4.5 radians downstream of the source. It was not possible to obtain corresponding data for the large-scale experiments by viewing through the sidewall windows of the Coriolis Facility, as the dye in the current became too diluted at the interface between the current and the ambient fluid, making it impossible to define the current boundary reliably by visual inspection.

Figure 11 shows the along-wall height data obtained in the small-scale facility. The figure displays the relative current height $h(x) / h_{m}$ as a function of the nondimensional distance $x /\left(2 \pi R_{S}\right)$ from the source $\left(R_{S}\right.$, the radius of the small tank, is $0.4475 \mathrm{~m}$ ). The four data points correspond to measurements at the four reference locations. Each data point represents an average of the 66 measurements for the individual experiments. In each case the data were collected at an instant just before the end of the experiment, i.e. when the head of the current had propagated almost around the entire circumference of the tank. The figure shows that the current height at the wall decreases linearly over a large interval of the current, which extends at least over the region $0.24 \leqslant x /\left(2 \pi R_{s}\right) \leqslant 0.73$. In $\S \S 4.2$ and 4.3 it was found that the currents are faster than predicted by the theory, during the early stages of an experiment. This initial increased speed is a consequence of the decrease in the current height along the wall of the tank. 
Rotating gravity currents: small-scale and large-scale experiments and a model 51

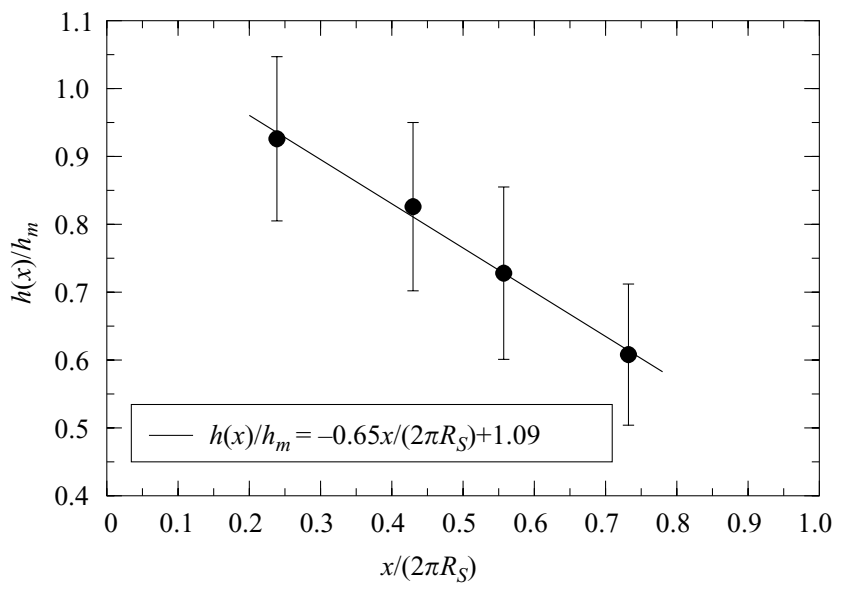

FIGURE 11. The relative current height, $h(x) / h_{m}$, for the small-scale experiments as a function of the non-dimensional distance $x /\left(2 \pi R_{S}\right)$ from the source.

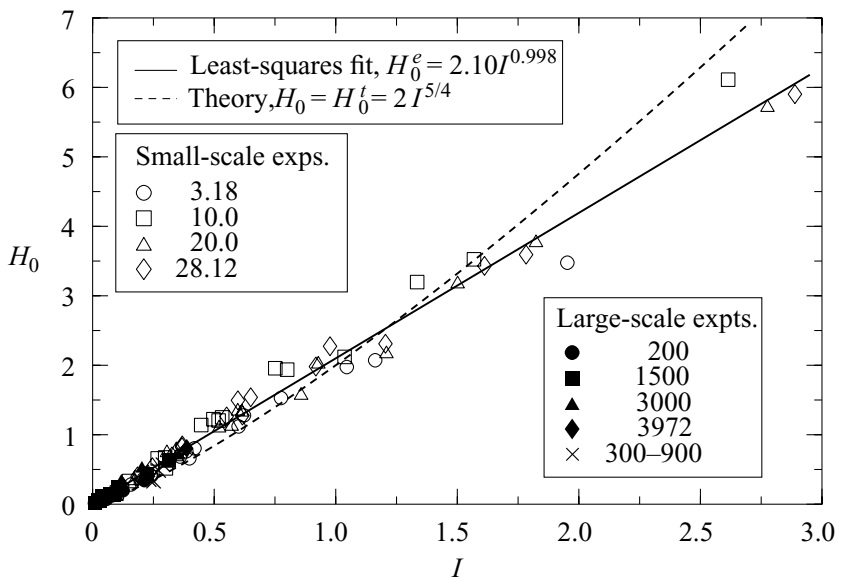

FIGURE 12. Non-dimensional maximum current height near the source, $H_{0}=h_{m} / R_{D}$, as a function of $I$. The numbers in the key are the values of $q_{0}$ in $\mathrm{cm}^{3} \mathrm{~s}^{-1}$.

\subsubsection{Scaling of the current height with the parameter I}

In order to facilitate a comparison between the experiments and the theoretical model of $\S 3$, the measured maximum current height $h_{m}$ is identified with the current height $h_{0}$ predicted by (3.15). Figure 12 gives a summary of the measured values for $h_{m}$ in non-dimensional form, in terms of $H_{0}=h_{m} / R_{D}$ as a function of $I$. (Recall that the current depths predicted by our present model, that predicted by the model of Avicola \& Huq (2002) and that defined by Horner-Devine et al. (2006) are the same.)

A least-squares fit to the data points for the small-scale and large-scale experiments gives

$$
H_{0}^{e}=2.10 I^{0.998},
$$

and is shown as the solid line in figure 12. The dashed line is the theoretical prediction $H_{0}=2 I^{5 / 4}$ of (3.22). It can be seen that experiment and theory are overall in good agreement; there is only a slight discrepancy between (3.22) and (4.1). 


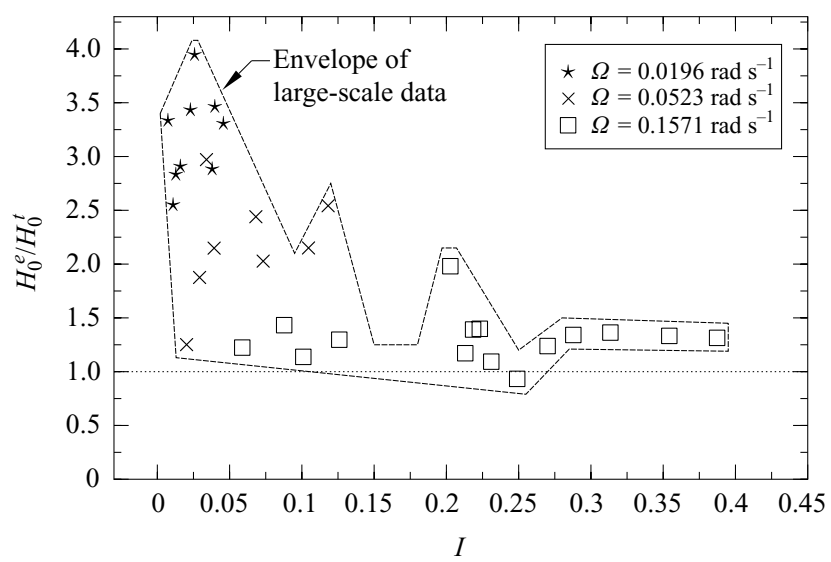

FIGURE 13. The ratio of measured current height, $H_{0}^{e}$, and theoretical height, $H_{0}^{t}$, given by (3.22) for the large-scale experiments as a function of $I$.

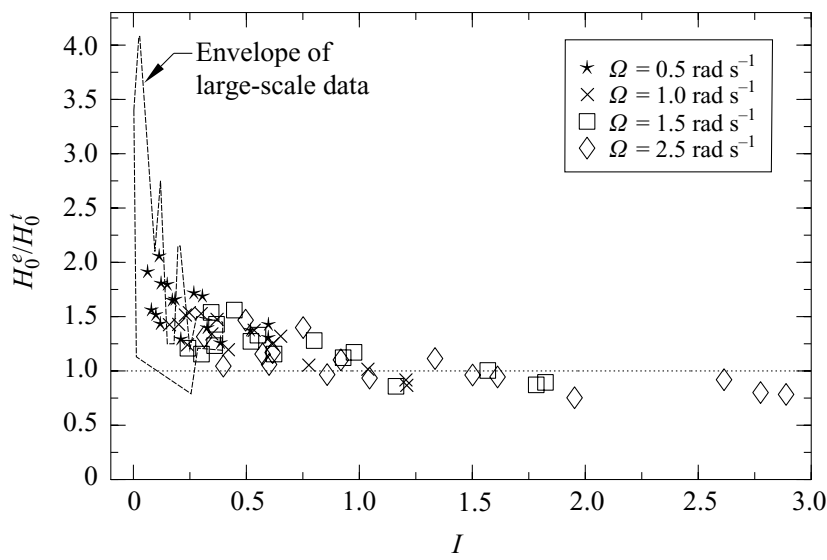

FIGURE 14. The ratio of measured current height, $H_{0}^{e}$, and theoretical height, $H_{0}^{t}$, as given by (3.22) for the small-scale experiments as a function of $I$.

Figures 13 and 14 identify the particular experiments responsible for this discrepancy between experiment and theory. The two figures display the ratio of the measured non-dimensional height $H_{0}^{e}$ and the non-dimensional predicted height $H_{0}^{t}$ as a function of the non-dimensional parameter $I$. The data for the large-scale experiments are shown in figure 13 while those for the small-scale experiments are shown in figure 14. For good agreement between experiment and theory, $H_{0}^{e} / H_{0}^{t}=1$. In both figures the data for different values of the rotation rate $\Omega$ are identified by different types of marker. In order to facilitate an easy comparison between the data for the large-scale and the small-scale experiments in these figures an envelope has been drawn around the large-scale data of figure 13 and this envelope has been superimposed onto figure 14.

Figures 13 and 14 reveal that for both large-scale and small-scale experiments the agreement between experiment and theory improves substantially with increasing values of the parameter $I$. For very low values, $I<0.05$, the experimentally measured value $H_{0}^{e}$ exceeds the predicted height $H_{0}^{t}$ by as much as $400 \%$ while agreement between experiment and theory is generally very good for $0.15<I<3$. 0 . The data 


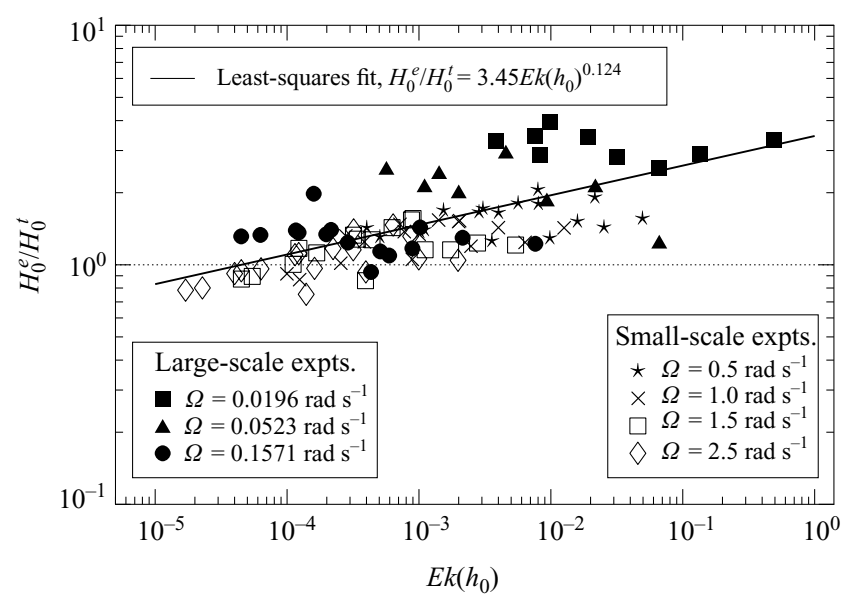

FiguRE 15. The ratio of the measured current height, $H_{0}^{e}$, and the theoretical height, $H_{0}^{t}$, given by (3.22) as a function of the Ekman number, $E k\left(h_{0}\right)$, defined by (3.30). A summary of all the data for the large-scale and small-scale experiments is shown.

also show, in particular, that in both sets of experiments agreement with theory is very good for runs with higher rotation rates while it is less favourable for the lower rotation rates. This suggests that currents with low values of $I$ are not governed by geostrophic balance and, consequently, cannot be described accurately by our model. It is these data points for low values of $I$ coupled with low rotation rates which are responsible for the deviation of the least-squares fit (4.1) from the predictions of our model in (3.15) and (3.22).

Inspection of (3.23) reveals that experiments with low values of $I$ are those for which the discharge rate $q_{0}$ is small and for which the reduced gravity $g^{\prime}$ is large. Reference to (3.15) shows that these currents are expected to be shallow. Hence, it is to be expected that the motion of currents with decreasing values of $I$ will be subject to increasing viscous effects; this is verified in the following section.

\subsubsection{Scaling of the current height with the Ekman number}

The Ekman number $E k\left(h_{0}\right)$ based on the current height $h_{0}$ of (3.15) is defined by (3.30). Figure 15 displays the ratio of the measured current height $H_{0}^{e}$ and the theoretical height $H_{0}^{t}$ as a function of $E k\left(h_{0}\right)$. The summary of all the data for the large-scale and small-scale experiments is shown. The figure reveals that agreement between experiment and theory is best for the smallest Ekman numbers, i.e when viscous effects are negligible. Agreement deteriorates with increasing Ekman number, i.e. when viscous effects become important. A comparison of the scalings for $\Omega, q_{0}$ and $g^{\prime}$ in (3.23) and (3.30) reveals that the smallest values of $I$ correspond to the largest values of $E k\left(h_{0}\right)$. Hence, the data points at higher values of $E k\left(h_{0}\right)$ in figure 15 correspond to data points with lower values of $I$ in figures 13 and 14 .

The data in figure 15 collapse onto a single straight line. Hence, the ratio $H_{0}^{e} / H_{0}^{t}$ scales with the Ekman number $E k\left(h_{0}\right)$. The solid line is a power-law least-squares fit given by

$$
\frac{H_{0}^{e}}{H_{0}^{t}}=3.45 E k\left(h_{0}\right)^{0.124}
$$




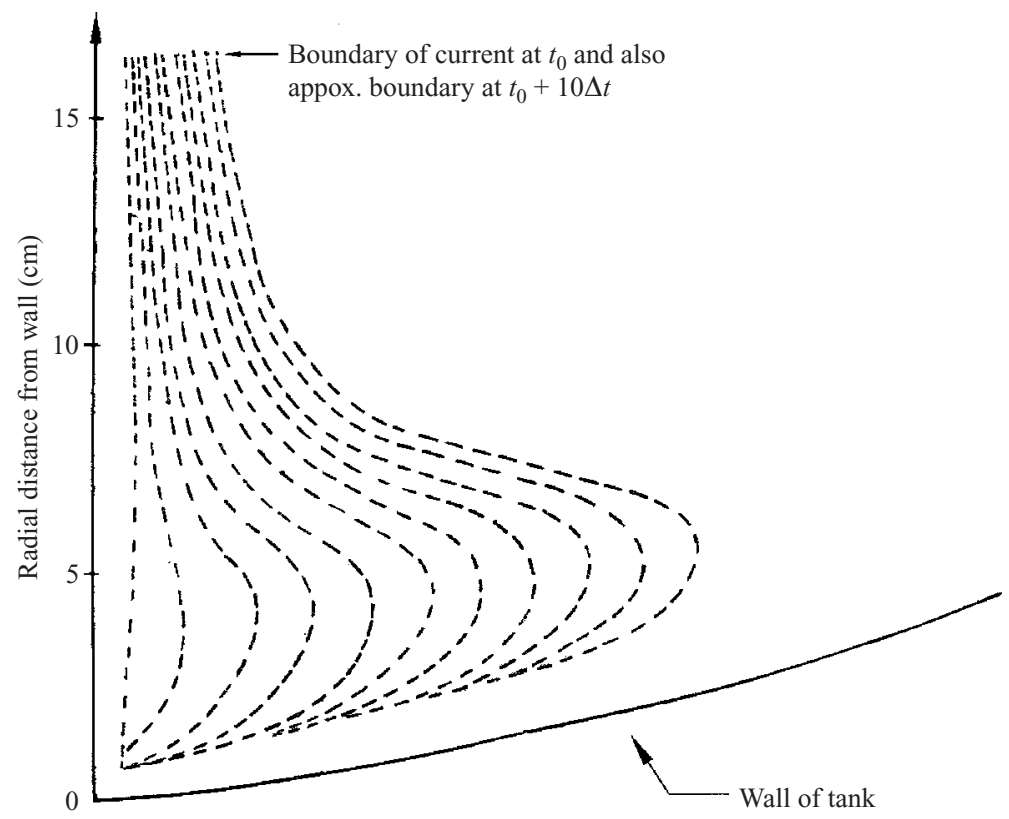

FIGURE 16. Typical surface-velocity profiles obtained from the observation of small tracer particles advected downstream with a current in the small-scale facility. The profiles were collected at 1.5 radians, corresponding to $67 \mathrm{~cm}$ downstream of the source. The experimental conditions are $\Omega=1.0 \mathrm{rad} \mathrm{s}^{-1}, g^{\prime}=16 \mathrm{~cm} \mathrm{~s}^{-2}, q_{0}=20 \mathrm{~cm}^{3} \mathrm{~s}^{-1}$ yielding, $I=0.34$. The time interval $\Delta t$ between two successive profiles is $0.5 \mathrm{~s}$.

\subsection{Current width}

\subsubsection{Introductory remarks}

Measuring the position of the current head and the current height is straightforward, as seen in $\S \S 4.2$ and 4.4. However, measurement of the current width is more involved since the edge of the current seen on the fluid surface is qualitatively different depending the value of $I$; this dependence was discussed in $\S 4.1 .1$.

In particular, for currents with low values of $I$ the width identified and seen on the fluid surface by the outline of the dyed fluid does not represent the current width relevant to the dynamics of the geostrophic problem. This question is addressed by considering the region where the bulk of the fluid motion in the current takes place. To identify this region surface-velocity profiles for the currents were obtained.

\subsubsection{Surface-velocity profiles}

The surface velocity of the currents was determined by measuring the motion of small tracer particles floating on the surface of the current. The tracer particles were dropped onto the fluid surface in a straight line radially across the current. The particles were then advected downstream with the local surface-flow velocity.

An example showing typical surface-velocity profiles obtained from a small-scale experiment with $I=0.3449$, a value similar to that of the current shown in figure $3(b)$, is displayed in figure 16. The figure shows ten profiles collected after successive 0.5 second intervals. The boundary of the current indicated in figure 16 corresponds to the visible boundary between the dyed current fluid, and the clear ambient fluid as seen in figures 3 and 4 . The width of the visible boundary is essentially constant during the short observation period of 5 seconds. Figure 16 reveals that dye visualizations would suggest a current width of approximately $16 \mathrm{~cm}$ for the example shown. However, the 

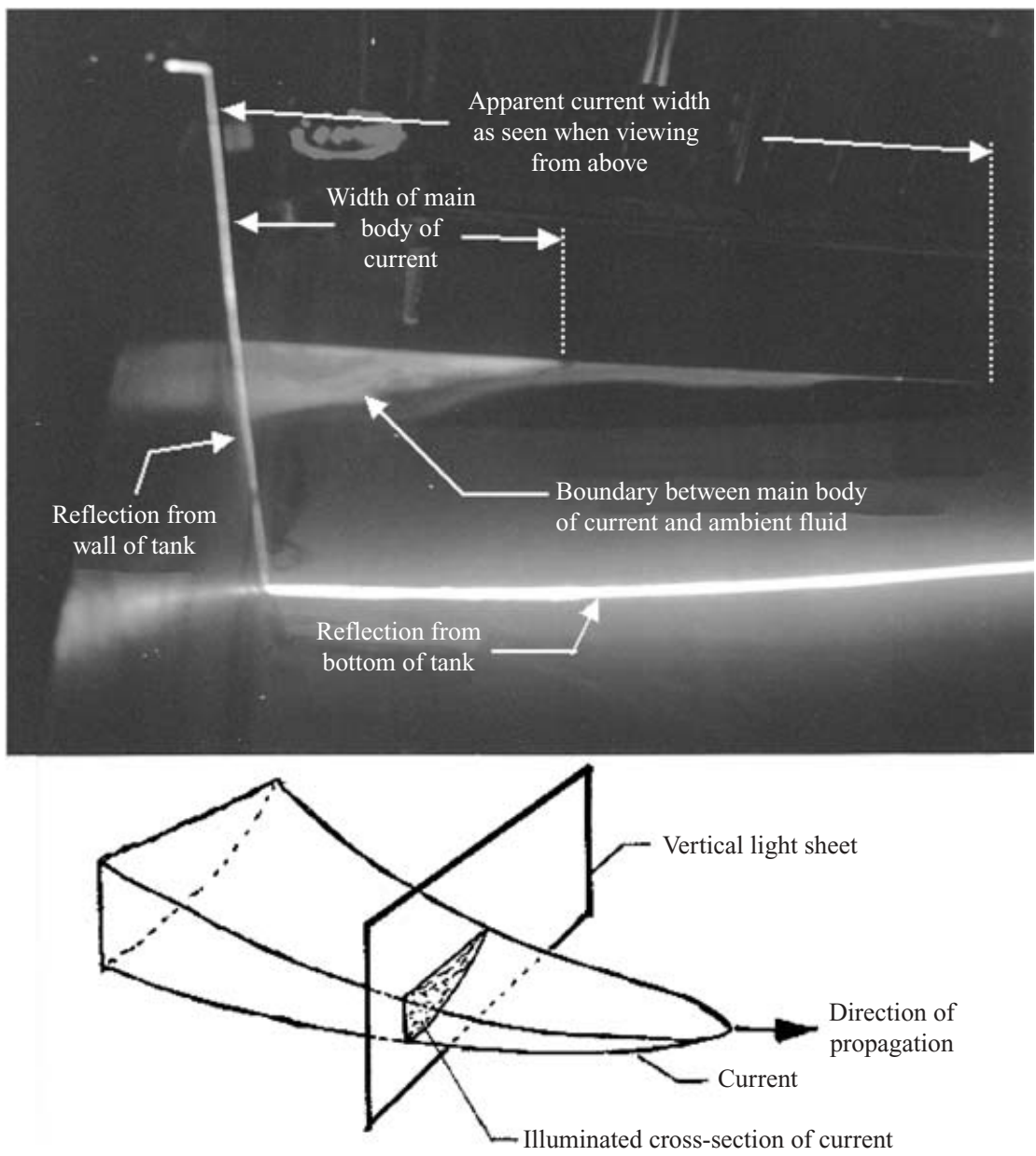

FiguRE 17. Photograph of the cross-section (in a $(y, z)$-plane; see figure 2) of a large-scale current illuminated by a vertical light sheet, as illustrated in the diagram below the photograph. The photograph was taken at approximately 0.844 radians, corresponding to $5.488 \mathrm{~m}$, downstream of the source during an experiment with $\Omega=0.1571 \mathrm{rad} \mathrm{s}^{-1}, g^{\prime}=8.5 \mathrm{~cm} \mathrm{~s}^{-2}$, $q_{0}=3000 \mathrm{~cm}^{3} \mathrm{~s}^{-1}$ and $I=0.216$.

velocity profiles illustrate that the flow velocity is largest in a substantially narrower near-wall zone which is only about $8-10 \mathrm{~cm}$ across. In this near-wall zone the current is also deepest and, hence, this is the region where the bulk of the fluid motion takes place. Consequently, the width of the near-wall zone is the appropriate measure of the current width for the flow dynamics.

\subsubsection{Cross-sectional illumination of currents}

The discussion in $\S 4.5 .2$ illustrated that dye-visualization experiments overestimate the current width relevant to the problem. This observation is further substantiated by visualizations of the current cross-section in a $(y, z)$-plane; see figure 2 . The photograph in figure 17 displays such a visualization for a current in a large-scale experiment with $I=0.216$. For this visualization the fluid released at the source contained sodium fluorescein. A vertical light sheet intersected the current as illustrated below the photograph. The light sheet was positioned at 0.844 radians, corresponding to $5.488 \mathrm{~m}$ downstream of the source. The bright near vertical line in the left-hand part 
of the photograph is due to light reflected from the wall of the tank; the very bright horizontal and slightly curved line in the lower half of the photograph represents light reflected from the bottom of the tank. The $y$-coordinate of figure 2 corresponds approximately to the direction horizontally across the page.

Figure 17 shows that the current is deepest at the wall of the tank, as expected from (3.10). We have not attempted quantitative comparisons between the parabolic profile given by (3.10) and visualizations such as that in figure 17. The photograph was taken by a camera positioned a few centimetres above the fluid surface and angled slightly downwards. Consequently, the cross-section as it appears in figure 17 is slightly distorted.

Again, figure 17 suggests that the width of the main current is substantially smaller than the apparent total width identified by the fluorescein illumination. There is a clear boundary between the fluid which constitutes the main body of the current and the adjacent fluid contaminated by fluorescein. This boundary is only seen on visualizations of the cross-sections and is not visible when viewing the current from above, as in figures $3(a-c)$ or figure 4. This corroborates the conclusion of $\S 4.5 .2$ that data for the current width based on measuring the width seen on the liquid surface in dye-visualization experiments give substantial overestimates. For example, the main current shown in figure 17 is only about half as wide as the total apparent width suggested by the fluorescein illumination. This is consistent with the discussion of figure 16 in $\S 4.5 .2$ concerning how the surface-velocity profiles for a current with a similarly low value of $I$ suggest that the main current is only about half as wide as the width indicated by the outline of the food colouring. The shallow surface layer outside the main body of the current is fluid in the surface Ekman layer produced by the (small) stress at the surface.

\subsubsection{Error estimates for the measurement of the current width}

For currents with low to intermediate values of $I$ (figures $3 a$ and $3 b$ ), measurements of the width of the near-wall zone discussed in $\S 4.5 .2$ were used for comparison with our model. Figure 16 shows that the transition between the near-wall zone and the outer current zone is gradual. Hence, the definition of the width of the near-wall zone involves a degree of ambiguity. Typical maximum errors resulting from this uncertainty for the measurement of the current width from surface-velocity profiles are estimated to be no larger than $10 \%-15 \%$.

For low values of $I$ (figure $3 a$ ) the current width was always measured by considering surface-velocity profiles only. However, for intermediate values of $I$ (figure $3 b$ ) an additional second measurement was taken. For these currents the experiments revealed that the narrowest width, at the contraction where the water from the gyre begins its motion along the wall, agrees well with the width obtained from surface-velocity profiles. Because of this we measured both quantities for currents with intermediate values of $I$ and we then used their average as the current width for comparison with the theory.

For high values of $I$ one obtains the type of current shown in figure 3(c). For these currents it is straightforward to measure the appropriate current width, which is simply the width just downstream of the gyre. The currents with high values of $I$ in the small-scale facility are typically of the order of only $10-20 \mathrm{~mm}$ across. The absolute measurement error for the width of the small-scale currents was of the order of $\pm 4 \mathrm{~mm}$, which corresponds to relative errors of at least $20 \%-40 \%$. Corresponding errors for currents with higher values of $I$, in the large-scale facility, are estimated to be of the order of $10 \%$. 


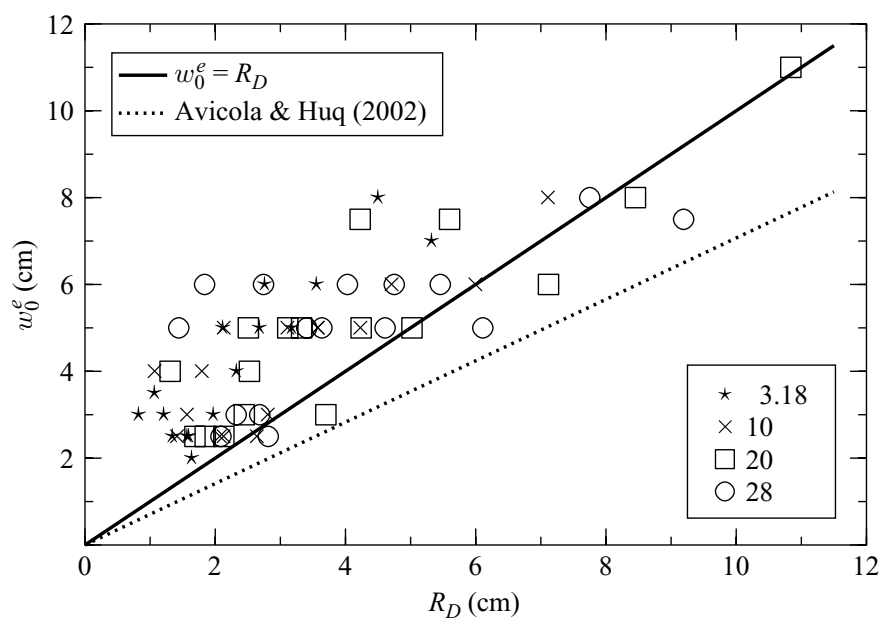

FIGURE 18. The measured current width, $w_{0}^{e}$, as a function of the Rossby deformation scale $R_{D}$, for the small-scale experiments. The numbers in the key are the values of $q_{0}$ in $\mathrm{cm}^{3} \mathrm{~s}^{-1}$.

At high values of $I$ there is another mechanism which can result in the data being biased towards an overestimation the current width. At high values of $I$, i.e. when $g^{\prime}$ is small while $q_{0}$ and $\Omega$ are large, the currents are deep and fast moving. For such currents, shear instabilities appear on the boundary between current fluid and ambient fluid. These instabilities can be seen in figure $3(c)$ in the region $0.2-1.0$ radians downstream (two to ten sectors of the reference grid) of the source. The figure reveals that the amplitudes of these instabilities are of the order of up to 10$20 \mathrm{~mm}$. This translates into $100 \%-200 \%$ of the total width of these narrow currents in the small-scale facility. Together with the absolute measurement error, the total relative measurement error can lie within roughly $100 \%-300 \%$ for the currents with the highest values of $I$. The corresponding relative error for large-scale currents is substantially smaller. The magnitude of the amplitudes of the shear instabilities for currents with highest values of $I$ in the large-scale facility is of the same magnitude as in the small-scale facility. However, the currents in the large-scale facility are typically at least about $200 \mathrm{~mm}$ across. Hence, the relative error associated with with the shear instabilities is at least one order of magnitude smaller for the large-scale currents than for the small-scale currents.

\subsubsection{Scaling of the current width with the parameter I}

Figure 18 displays the experimental data for the current width measured in the small-scale experiments. The theoretical model in $\S 4$ yields $W=w_{0}^{e} / R_{D}=1$. Hence, good agreement between experiments and theory requires $w_{0}^{e}=R_{D}$, identified by the solid line in figure 18. The figure reveals satisfactory agreement between experiment and theory. The dotted line in figure 18 represents the theoretical prediction for the current width from the model of Avicola \& Huq (2002), which is given by $w_{0}=(1 / \sqrt{2})\left(g^{\prime} q_{0} / \Omega^{3}\right)^{1 / 4}$ in our nomenclature. Figure 18 shows that the present model describes the experimental data slightly better than that of Avicola \& Huq (2002).

Figure 19 shows the ratio $w_{0}^{e} / R_{D}$ as a function of the non-dimensional parameter $I$ for the small-scale experiments. The figure suggests that the agreement between experiment and theory generally tends to be better at lower values of $I$ than at higher ones. As discussed in $\S 4.5 .4$, currents with high $I$ develop shear instabilities at 


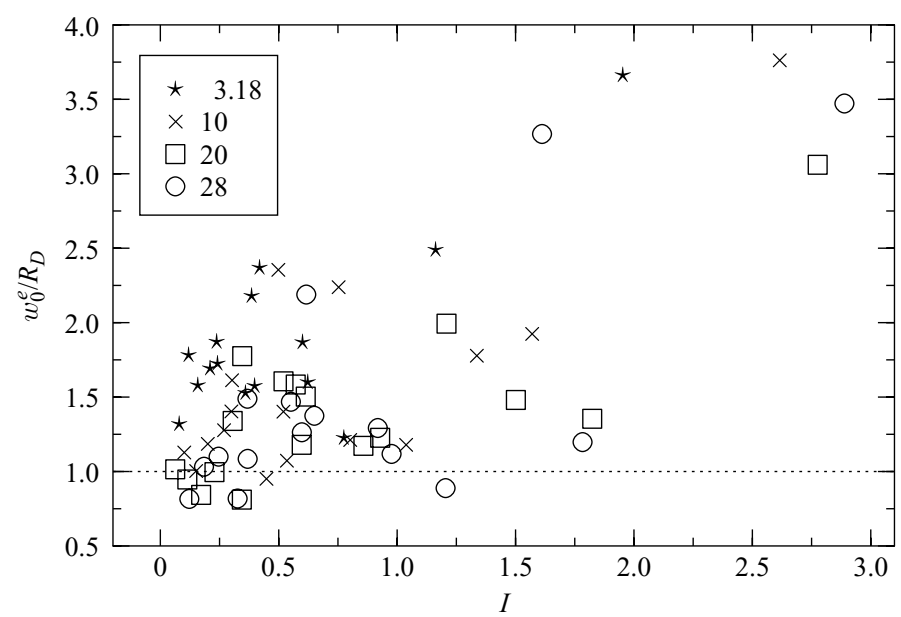

FIGURE 19. The ratio of the measured and predicted current widths, $w_{0}^{e} / R_{D}$, as a function of $I$ for the small-scale experiments. The numbers in the key are the values of $q_{0}$ in $\mathrm{cm}^{3} \mathrm{~s}^{-1}$.

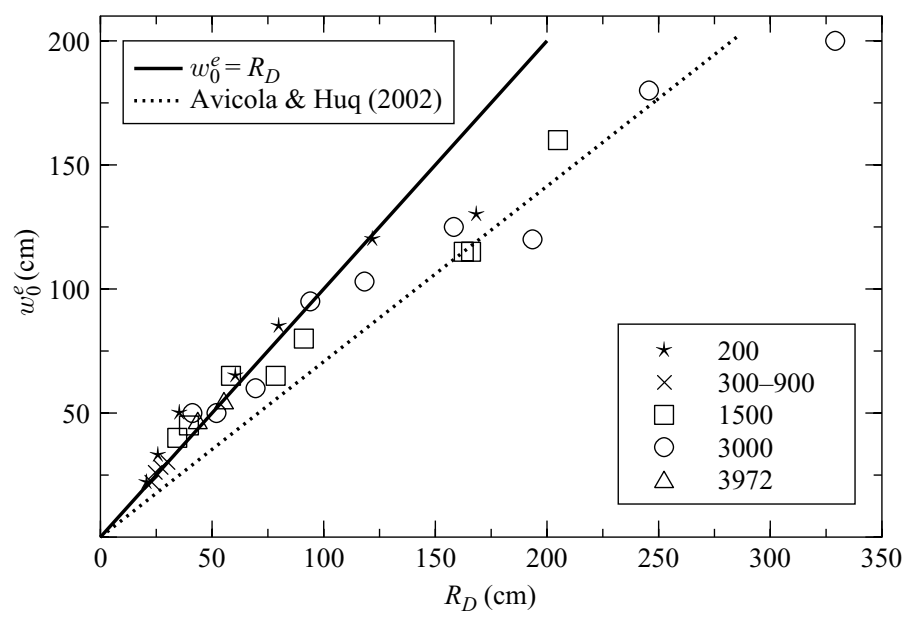

FIGURE 20. The measured non-dimensional current width, $w_{0}^{e}$, as a function of the Rossby deformation scale, $R_{D}$, for the large-scale experiments. The numbers in the key are the values of $q_{0}$ in $\mathrm{cm}^{3} \mathrm{~s}^{-1}$.

the interface between current fluid and ambient fluid and it was seen that these can introduce relative errors of order $100 \%-300 \%$ for the narrowest currents at highest values of $I$ in the small-scale facility. These large relative errors probably explain why agreement between experiment and theory in figure 19 is generally better at lower values of $I$ rather than at higher ones.

Figure 20 displays the experimental data for the current width measured in the large-scale experiments in a graph corresponding to that of figure 18 for the smallscale experiments. The figure reveals that the agreement between experiment and theory for the large-scale experiments is very good for currents with maximum widths up to about $1.2 \mathrm{~m}$ but that it becomes somewhat less favourable for wider currents. Corresponding to figure 18, the dotted line in figure 20 represents the theoretical prediction for the current width from the model of Avicola \& Huq (2002). For 


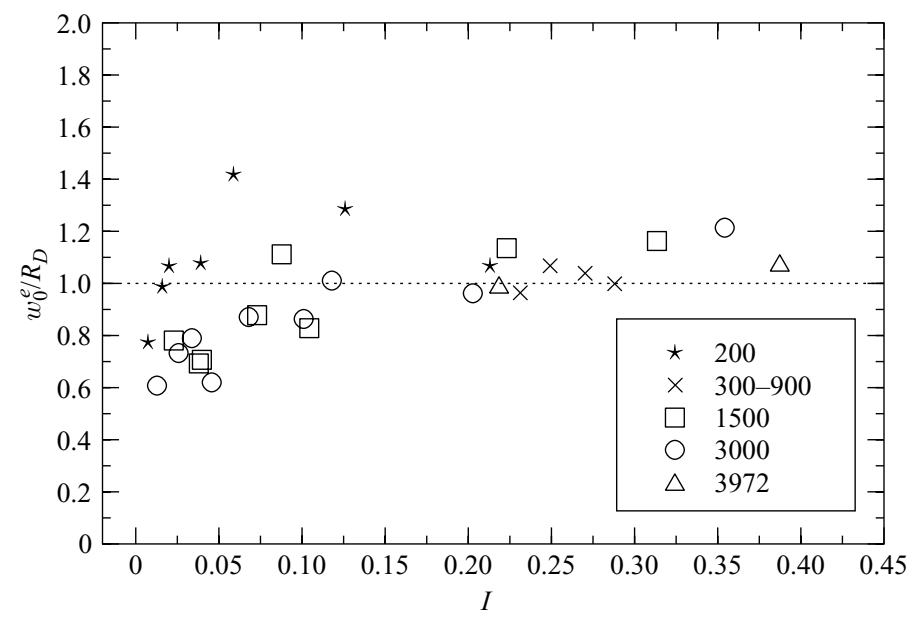

FIGURE 21. The ratio of the measured and predicted current widths, $w_{0}^{e} / R_{D}$, as a function of $I$ for the large-scale experiments. The numbers in the key are the values of $q_{0}$ in $\mathrm{cm}^{3} \mathrm{~s}^{-1}$.

narrower currents, figure 20 shows that the present model describes the experimental data slightly better than the model of Avicola \& Huq (2002). For very wide currents, with $w_{0}^{e} \geqslant 150 \mathrm{~cm}$, our model overpredicts the experimental data and the prediction of Avicola \& Huq (2002) appears to describe the experiments somewhat better.

Figure 21 shows the ratio $w_{0}^{e} / R_{D}$ as a function of the non-dimensional parameter $I$ for the large-scale experiments. The data show that the quality of the agreement between experiment and theory is, essentially, the same over the entire interval $0 \leqslant I \leqslant 0.4$ for which large-scale experiments were carried out. However, the data scatter is somewhat higher for smaller than for higher values of $I$.

Figure 22 shows a summary of all data points for $w_{0}^{e} / R_{D}$ for the small-scale and the large-scale experiments. The figure reveals that agreement is overall most favourable for the large-scale experiments with low values of $I$. This observation can be understood in terms of the measurement errors discussed in $\S 4.5 .2$. For small-scale experiments at high values of $I$ the shear instabilities at the current boundary lead to very large relative errors. This is reflected by the data in figure 22 and this source of error cannot be eliminated from the measurements. At lower values of $I$, when currents are generally slow, errors from the shear instabilities are no longer significant. The main source of error is now that associated with current fluid spilling out of the main current and contaminating the ambient fluid, as discussed in connection with figures 16 and 17 . However, this source of error can be largely suppressed for currents with low to intermediate values of $I$ if one measures the current width, as discussed in $\S 4.5 .2$. This leads to smaller errors and, hence, better agreement between experiment and theory for low values of $I$.

\subsubsection{Scaling of the current width with the Reynolds number}

Figure 23 displays the summary of all data for $w_{0}^{e} / R_{D}$ from the large-scale and the small-scale experiments as a function of $\operatorname{Re}\left(w_{0}, u_{0}\right)$, defined in (3.29). The data points below about $\operatorname{Re}\left(w_{0}, u_{0}\right)=5 \times 10^{3}$ are those for the small-scale experiments while those above this value are from the large-scale experiments.

Figure 23 reveals that the best agreement between experiment and theory is in the approximate range $10^{3} \leqslant \operatorname{Re}\left(w_{0}, u_{0}\right) \leqslant 5 \times 10^{4}$. The deterioration in agreement for 


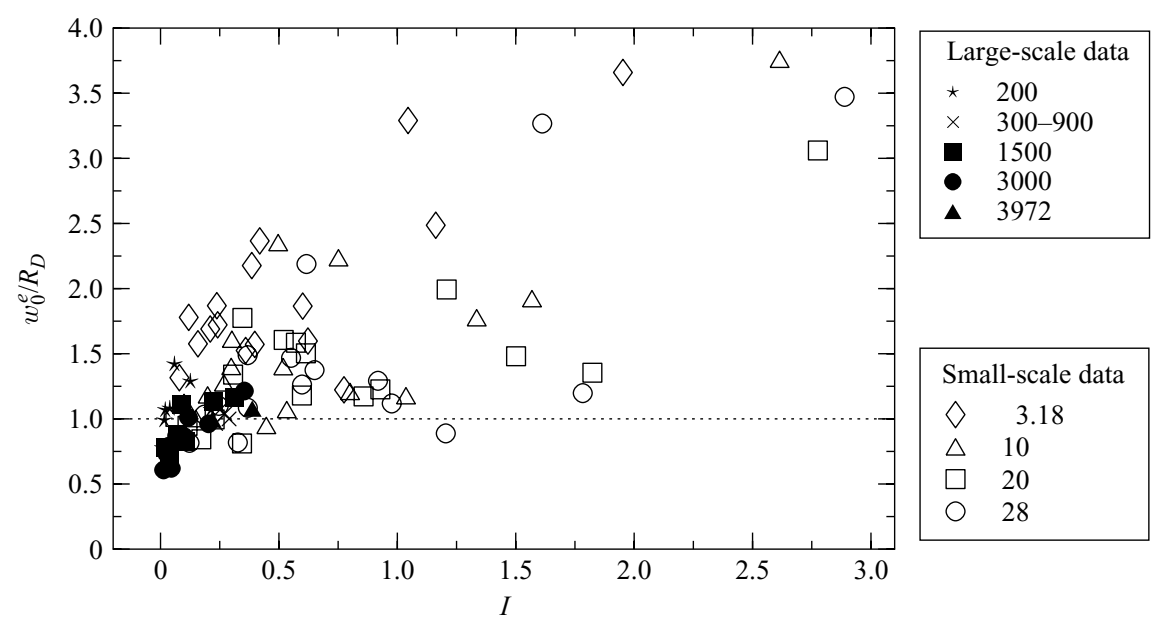

FIGURE 22. Summary of all small-scale and large-scale data for the ratio of the measured and predicted current widths, $w_{0}^{e} / R_{D}$, as a function of $I$. The numbers in the key are the values of $q_{0}$ in $\mathrm{cm}^{3} \mathrm{~s}^{-1}$.

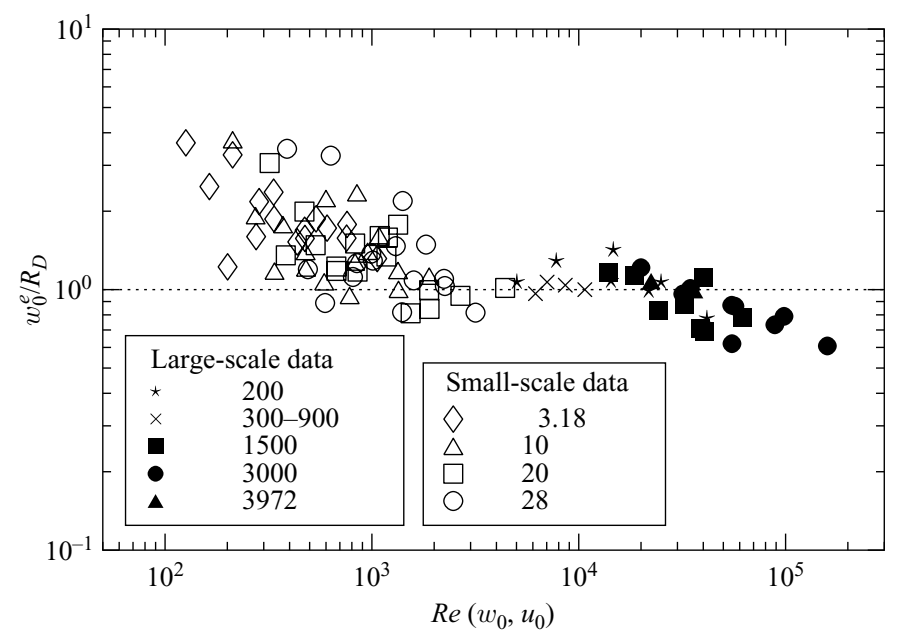

FIGURE 23. Summary of all small-scale and large-scale data for the ratio of the measured and predicted current widths, $w_{0}^{e} / R_{D}$, as a function of the Reynolds number $\operatorname{Re}\left(w_{0}, u_{0}\right)$. The numbers in the key are the values of $q_{0}$ in $\mathrm{cm}^{3} \mathrm{~s}^{-1}$.

$\operatorname{Re}\left(w_{0}, u_{0}\right) \leqslant 10^{3}$ again reflects measurement errors. It does not reflect an increased importance of viscous effects in comparison with the predictions of our inviscid model. Comparison of figures 23 and 22 shows that the data points for $\operatorname{Re}\left(w_{0}, u_{0}\right) \leqslant 10^{3}$ correspond to those with large values of $I$. These points are subject to large relative measurement errors arising in association with the shear instabilities at the boundary of the currents.

Figure 23 further indicates that agreement between experiment and theory for the current width may deteriorate for the large-scale currents at higher Reynolds numbers, roughly above $\operatorname{Re}\left(w_{0}, u_{0}\right)=5 \times 10^{4}$. However, this observation is based on a relatively small number of data points and may not be significant. The behaviour 
cannot be reflecting increased turbulent mixing of current fluid and ambient fluid at their interface for high Reynolds numbers; turbulent mixing would tend to result in overestimates for the experimentally measured current width $w_{0}^{e}$. Consequently one would expect $w_{0}^{e} / R_{D}>1$, rather than $w_{0}^{e} / R_{D}<1$ as displayed by the data in the figure.

When viscous effects are important, the fluid on the current surface is no longer governed by geostrophy. As a consequence this fluid can leak out of the main current. This results in the current width seen on the fluid surface being greater than the width relevant to the current dynamics. The current width is then no longer constant because fluid draining out of the current spreads across the surface. The current width as seen on the fluid surface thus becomes a function of time. Our observations have shown that this is the case for low to intermediate values of $I$, i.e. for the two current types shown in figures 3(a) and 3(b). As discussed earlier, we measured the growth of the current width identified by the dye visualization experiments on the fluid surface, as a function of time at various reference locations $\mathrm{RL}_{i}$ (figure 10) distributed along the wall of the tanks of the small-scale and the large-scale facilities. The data analysis showed that the surface width at each reference location $\mathrm{RL}_{i}$ grows roughly as, $\left(t^{*}\right)^{0.5}$ where $t^{*}=t-t_{R L}$ and $t_{R L}$ is the time of arrival of the current head at reference location $\mathrm{RL}_{i}$. The result $\left(t^{*}\right)^{0.5}$ confirms the corresponding measurements of Lentz \& Helfrich (2002, p. 264). In $\S \S 4.2$ and 4.3 it was observed that the currents slow down during the later stages of each experiment. This deceleration is a consequence of the leakage of fluid out of the main current. When fluid leaks out of the current the assumption of mass conservation for the main current in (3.13) and (3.17) is no longer valid.

\section{Froude number}

In $\S 4.3$ it was observed that the velocity of the current head decreases with time $T$.

We have calculated experimental values for the Froude number, $\operatorname{Fr}\left(T_{i}\right)=\overline{u_{0}^{e}} / \sqrt{g^{\prime} h_{m}}$, based on the measured current mean velocity $\overline{u_{0}^{e}}=l_{i} / t_{i}$ and the measured maximum depth $h_{m}$ near the source. The results obtained in this way are displayed in figures 24(a) and $24(b)$ for the small-scale and the large-scale experiments, respectively. The figures reveal that, for both sets of experiments, the Froude number is in the approximate range $0.1 \leqslant F r \leqslant 1.51$, and they illustrate how $F r$ decreases with time $T$. For the majority of runs the Froude number had value just below a 0.8 at the beginning of the experiment. This is reflected in the two figures through the very high concentrations of data points around $T=0, F r=0.8$.

In these figures the data points for the different runs have been grouped into three different sets according to their values of the parameter $I$. Both figures reveal that there is a general trend for the Froude number to adopt lower values for smaller values of $I$. Low values of $I$ correspond to high values of the Ekman number and, hence, to the parameter regime where viscous effects become increasingly important and where our inviscid model is no longer valid.

For the vast majority of all data points for the highest values of $I$ (lowest $E k$ ) the Froude number lies within the interval $0.35 \leqslant F r \leqslant 0.78$. The solid lines, given by $F r=1.2 T^{-0.22}$ and $F r=0.85 T^{-0.09}$, interpolating the data points in figures $24(a)$ and $24(b)$ were determined by plotting all data in double-logarithmic representation and then fitting lines through the regions displaying the highest concentrations of data points by eye. These lines are meant to serve as a rough guideline summarizing the behaviour of the data for the higher values of the parameter $I$. It is emphasized that they do not represent least-squares interpolations. The dotted lines in figures 24(a) and $24(b)$ serve to facilitate an easy comparison between the data for the small-scale 

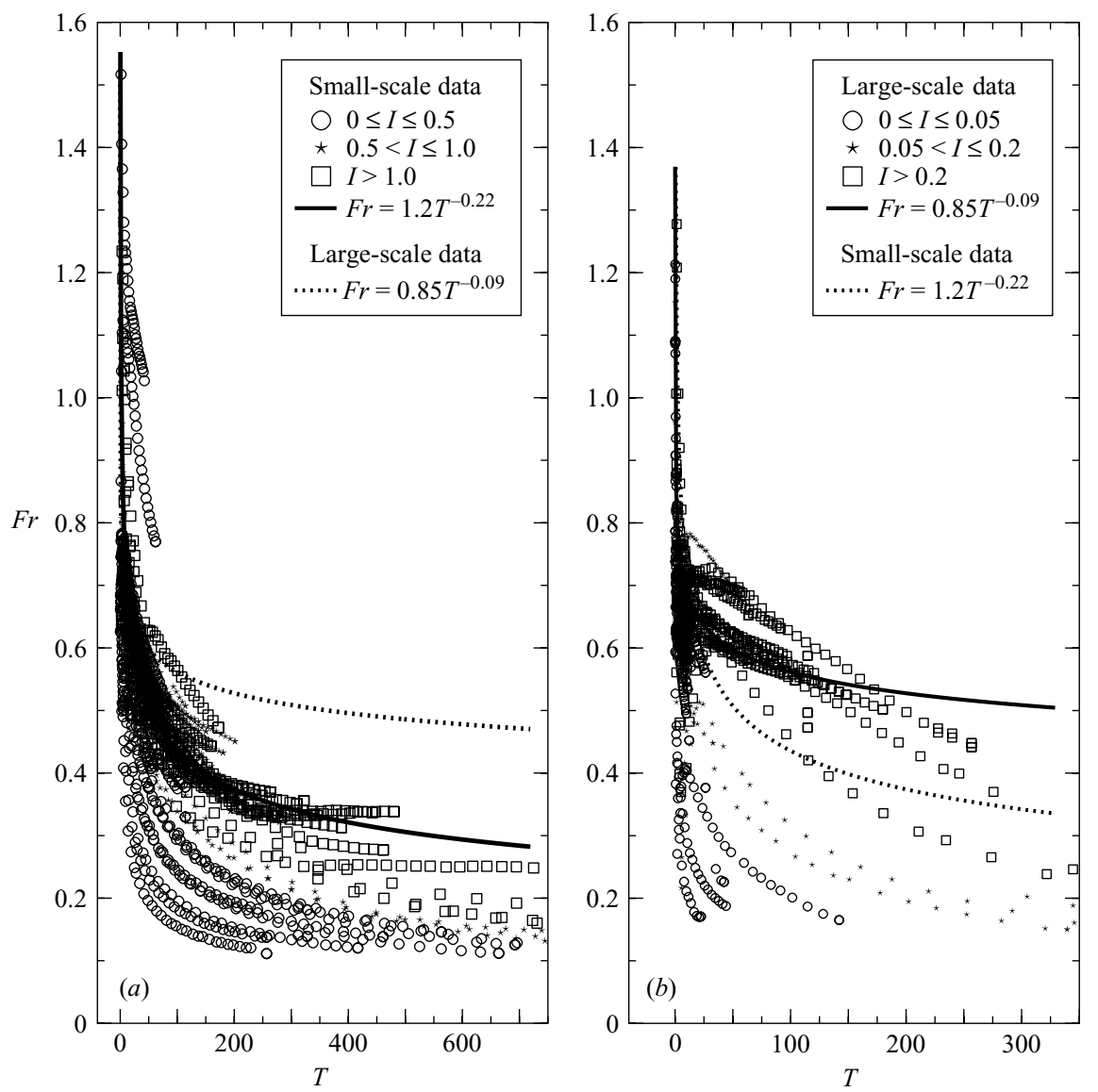

FIGURE 24. Froude number $F$ as a function of the non-dimensional time, $T$, for $(a)$ the small-scale experiments and $(b)$ the large-scale experiments.

and large-scale experiments. The dotted line in figure 24(a) corresponds to the solid line in figure 24(b) and vice versa.

Our experimental result, that the majority of data for the Froude number lie within $0.35 \leqslant F r \leqslant 0.78$, is overall in good agreement with the predicted value of $F r=0.5303$ (3.27). Our values for the Froude number are lower than the value $F r \approx \sqrt{2}=1.41$ obtained by Martin \& Lane-Serff (2005), who extended the theory of Benjamin (1968) to rotating currents, and they are also lower than the value $F r=1$ predicted by Shin et al. (2004). In comparison, the dam-break experiments of Griffiths \& Hopfinger (1983) suggested that $F r=1.3 \pm 0.2$ or $F r=1.15 \pm 0.1$, depending on whether the hydrostatic head $h_{u}$ or the depth $h_{n}$ of the current head was used to calculate the Froude number. Similarly, Stern, Whitehead \& Hua (1982) reported $F r=1.6$ for their experiments at highest Reynolds numbers; they had predicted the Froude number to be in the range $1.54 \leqslant F r \leqslant 1.58$. In $\S 3$ we found that $F r=1$ if one uses the expressions of Avicola \& Huq (2002) to calculate the Froude number corresponding to our expression (3.25). Since figures 24(a) and 24(b) reveal that the Froude number is just below 0.8 for the majority of experiments for small times $T$, our experimental data lie between our prediction and that of Avicola \& Huq (2002). At the later stages of the experiment the measured values of the Froude number more closely resemble 
Rotating gravity currents: small-scale and large-scale experiments and a model 63

the value predicted by our model. However, it should be emphasized that this does not imply that our model necessarily describes the experiments better at larger $T$ than that of Avicola \& Huq (2002). The reason for this is that the current slows down because of the drainage of fluid from the main current due to the growing bulge region near the source together with the additional drainage near the current surface identified in $\S 4.5 .6$. Because of this drainage the assumption of volume conservation underlying our model, and also the model of Avicola \& Huq (2002), is no longer valid.

\section{Summary and conclusions}

Laboratory simulations investigating the influence of the rotation of the earth on the dynamics of gravity-driven surface currents developing from fresh-water river discharges into the ocean have been described. Two complementary studies with experiments on substantially different spatial scales have been performed and the data collected in both studies have been compared with each other.

A simple geostrophic model for the flow was developed to predict the current height, width and velocity as a function of the relevant independent parameters governing the flow. The scalings obtained are the same as those previously deduced by Davies et al. (1993) and, more recently, by Lentz \& Helfrich (2002) on the basis of scaling arguments. However, since these authors used scaling arguments only, they were unable to give values for the constants missing from their proportionality relations. Our new model yields values for these constants and they have been compared with corresponding values obtained by Avicola \& Huq (2002) and Horner-Devine et al. (2006) wherever possible. Our theoretical analysis yielded a set of non-dimensional parameters suitable to summarize the measured data and to collapse the corresponding results from large-scale and small-scale experiments onto single lines. Our predictions are, generally, in good agreement with our experimental data. It is probably justified to conclude that overall they are in slightly better agreement with the experiments than the corresponding theoretical results of Avicola \& Huq (2002) and Horner-Devine et al. (2006).

For the derivation of our model we assumed that the flow is steady and that there are no variations along the current. However, the experiments have shown that this is not the case. Figure 11 revealed, for instance, that the current height decreases from the source to the current head. In this respect our model is, consequently, still incomplete. We presently do not have a definitive theoretical explanation why the current height decreases. Neither do we know whether it would be possible to incorporate these additional features into a refined version of our model which could still be solved analytically. Since the height predicted by our model is the same as that of Avicola \& Huq (2002) and Horner-Devine et al. (2006), the theoretical results of these authors are subject to the same discrepancies as our own in this respect. One of our referees pointed out that a downstream pressure gradient may be required to overcome the frictional resistance to the flow from the side wall and the fluid below. While this may well be the case, an investigation would necessitate finding numerical solutions of the flow problem, which was outside the scope of the present work.

For large rivers discharging into the ocean, the maximum volumetric-discharge rate $q_{0}$ is of the order of $10^{4} \mathrm{~m}^{3} \mathrm{~s}^{-1}$ and the maximum effective-rotation rate $\Omega$ arising from the rotation of the Earth is approximately $2 \pi / 86400 \mathrm{~s}^{-1}$. A typical value for the reduced gravity $g^{\prime}$ is $22 \mathrm{~cm} \mathrm{~s}^{-2}$. Using these values and $v=0.01 \mathrm{~cm}^{2} \mathrm{~s}^{-1}$ for the kinematic viscosity of water one determines an Ekman number $E k\left(h_{0}\right)=5.2 \times 10^{-4}$, a value $I=0.0011$ and a Reynolds number $\operatorname{Re}\left(w_{0}, u_{0}\right)=4.1 \times 10^{9}$. Comparison with 
figure 15 shows that the Ekman number for currents in the natural environment is of the order where very good agreement between experiment and theory for the current height is expected. This is the region where currents are governed by geostrophy and where the scaling relations from our model will hold. However, typical Reynolds numbers for currents in the natural environment are much larger than the maximum values, of about $\operatorname{Re}\left(w_{0}, u_{0}\right)=2 \times 10^{5}$, which can be reached even in the large-scale rotating tank. Figure 23 shows that it may be difficult to evaluate how accurately the results of our theoretical analysis and our experiments would predict the width of currents in nature. If, as is apparently indicated by figure 23 , agreement between experiment and theory would begin to deteriorate for $\operatorname{Re}\left(w_{0}, u_{0}\right)>5 \times 10^{5}$ then the results of even the largest possible laboratory experiments would not enable one to reliably extrapolate laboratory results for current width in the natural environment. However, currents in the natural environment are governed by geostrophy and, hence, it appears unlikely that they would not roughly follow the derived overall scalings for the current speed, depth and length. At present we do not have a definitive explanation why the agreement between experiment and theory for the current width in figure 23 seems to deteriorate slightly for larger Reynolds numbers. However, we believe that this may reflect an artifact arising in connection with the difficulties associated with measuring the current width accurately as discussed in $\S 4.5$.

The small-scale experiments were carried out as part of a study funded by the Natural Environment Research Council, UK. The large-scale experiments at the Coriolis Facility were funded by the European Union through an Access to Major Research Infrastructures grant. We would like to thank Dr Maria del Mar Flexas, Dr Farzam Zoueshtiagh and the staff of the Coriolis Facility for their help and assistance during the large-scale experiments.

\section{REFERENCES}

Avicola, G. \& HuQ, P. 2002 Scaling analysis for the interaction between a buoyant coastal current and the continental shelf: Experiments and observations. J. Phys. Oceanog. 32, 3233-3248.

Avicola, G. \& HuQ, P. 2003a The role of the outflow geometry in the formation of the recirculating bulge region in coastal buoyant outflows. J. Mar. Res. 61, 411-434.

Avicola, G. \& HuQ, P. $2003 b$ The characteristics of the recirculating bulge region in coastal buoyant outflows. J. Mar. Res. 61, 435-463.

Batteen, M. L. 1997 Wind-forced modelling studies of currents, meanders, and eddies in the Californian current system. J. Geophys. Res. 102, 985-1010.

Benjamin, T. B. 1968 Gravity currents and related phenomena. J. Fluid Mech. 31, 209-248.

Blumberg, A. F., Signell, R. P. \& Jenter, H. L. 1993 Modelling transport processes in the coastal ocean. J. Mar. Env. Engng 1, 31-52.

Bowman, M. J. \& Iverson, R. L. 1978 Estuarine and plume fronts. In Workshop on Oceanic Fronts in Coastal Processes, Marine Sciences Research Centre, Stony Brook, NY, May 25-27, Proc., pp. 87-104. Springer.

Boyer, D. L. \& DAvies, P. A. 2000 Laboratory studies of orographic effects in rotating and stratified flows. Annu. Rev. Fluid Mech. 32, 165-202.

Boyer, D. L., Haidvogel, D. B. \& Pérenne, N. 2001 Laboratory-numerical comparisons of flow over a coastal canyon. J. Atmos. Tech. 18, 1698-1718.

Chabert D’Hieres, G., Didelle, H. \& Obaton, D. 1991 A laboratory study of surface boundary currents: Application to the Algerian Current. J. Geophys. Res. 96, 12539-12548.

Davies, P. A., Jacobs, P. T. G. A. \& Mofor, L. A. 1993 A laboratory study of buoyant fresh water boundary currents in tidal crossflows. Oceanologica Acta 16, 489-503.

Griffiths, R. W. 1986 Gravity currents in rotating systems. Annu. Rev. Fluid Mech. 18, 59-89. 
Griffiths, R. W. \& Hopfinger, E. J. 1983 Gravity currents moving along a lateral boundary in a rotating fluid. J. Fluid Mech. 134, 357-399.

Griffiths, R. W. \& Linden, P. F. 1981 The stability of buoyancy-driven coastal currents. Dyn. Atmos. Oceans 5, 281-306.

HaCker, J. N. \& Linden, P. F. 2002 Gravity currents in rotating channels. Part 1. Steady-state theory. J. Fluid Mech. 457, 295-324.

Hickey, B. M., Pietrafesa, L. J., Jay, D. A. \& Boicourt, W. C. 1998 The Columbia River Plume Study: subtidal variability in the velocity and salinity fields. J. Geophys. Res. 103(C5), 10339-10368.

HogG, N. G. \& Johns, W. E. 1995 Western Boundary Currents, US National Report to International Union of Geodesy and Geophysics 1991-1994. Rev. Geophys., Supplement, 1311-1334.

Horner-Devine, A. R., Fong, D. A., Monismith, S. G. \& Maxworthy, T. 2006 Laboratory experiments simulating coastal river inflow, J. Fluid Mech. 555, 203-232.

Kawasaki, Y. \& Sugimoto, T. 1984 Experimental studies on the formation and degeneration processes of the Tsugaru warm gyre. In Ocean Hydrodynamics of the Japan and East China Sea (ed. T. Ichiye), pp. 225-238. D. Reidel.

Lentz, S. J. \& HeLfrich, K. R. 2002 Buoyant gravity currents along a sloping bottom in a rotating fluid. J. Fluid Mech. 464, 251-278.

Martin, J. R. \& Lane-SerfF, G. F. 2005 Rotating gravity currents. Part 1. Energy loss theory. J. Fluid Mech. 522, 35-62.

Martin, J. R., Smeed, D. A. \& Lane-SerfF, G. F. 2005 Rotating gravity currents. Part 2. Potential vorticity theory. J. Fluid Mech. 522, 69-89.

Münchow, A. \& GARvine, R. W. 1993a Buoyancy and wind forcing of a coastal current. J. Mar. Res. 51, 293-322.

MünChOW, A. \& GARvine, R. W. $1993 b$ Dynamical properties of a buoyancy-driven coastal current. J. Geophys. Res. 98(C11), 20063-20077.

NoF, D. \& Pichevin, T. 2001 The ballooning of outflows. J. Phys. Oceanogr. 31, 3045-3058.

RenNie, S., Largier, J. L. \& Lentz, S. J. 1999 Observations of low-salinity coastal current pulses downstream of Chesapeake Bay. J. Geophys. Res. 104(C8), 18227-18240.

Rivas, D., Velasco Fuentes, O. U. \& OchOA, J. 2005 Topographic effects on the dynamics of gravity currents in a rotating system. Dyn. Atmos. Oceans 39, 227-249.

Shin, J. O., Dalziel, S. B. \& Linden, P. F. 2004 Gravity currents produced by lock exchange. J. Fluid Mech. 521, 1-34.

Simpson, J. E. 1997 Gravity Currents in the Environment and the Laboratory, 2nd Edn. Cambridge University Press.

Stern, M. E., Whitehead, J. A. \& HuA, B.-L. 1982 The intrusion of a density current along the coast of a rotating fluid. J. Fluid Mech. 123, 237-266.

Thomas, P. J. \& Linden, P. F. 1998 A bi-modal structure imposed on gravity-driven boundary currents in rotating systems by effects of the bottom topography. Exps. Fluids 25, 388-391. 